IMSc-2007/11/14

\title{
Radiative Neutralino Production in Low Energy Supersymmetric Models
}

\author{
Rahul Basu ${ }^{1 *}$, P. N. Pandita ${ }^{2,3 \dagger}$ and Chandradew Sharma ${ }^{1 \ddagger}$ \\ ${ }^{1}$ The Institute of Mathematical Sciences, Chennai 600 113, India \\ 2 Department of Physics, North Eastern Hill University, Shillong 793 022, India Ind $^{\S}$ \\ ${ }^{3}$ The Abdus Salam International Centre for Theoretical Physics, Strada Costiera 11, 34014 Trieste, Italy
}

\begin{abstract}
We study the production of the lightest neutralinos in the radiative process $e^{+} e^{-} \rightarrow \tilde{\chi}_{1}^{0} \tilde{\chi}_{1}^{0} \gamma$ in low energy supersymmetric models for the International Linear Collider energies. This includes the minimal supersymmetric standard model as well as its extension with an additional chiral Higgs singlet superfield, the nonminimal supersymmetric standard model. We compare and contrast the dependence of the signal cross section on the parameters of the neutralino sector of the minimal and nonminimal supersymmetric standard model. We also consider the background to this process coming from the Standard Model process $e^{+} e^{-} \rightarrow \nu \bar{\nu} \gamma$, as well as from the radiative production of the scalar partners of the neutrinos (sneutrinos) $e^{+} e^{-} \rightarrow \tilde{\nu} \tilde{\nu}^{*} \gamma$, which can be a background to the radiative neutralino production when the sneutrinos decay invisibly. In low energy supersymmetric models radiative production of the lightest neutralinos may be the only channel to study supersymmetric partners of the Standard Model particles at the first stage of a linear collider, since heavier neutralinos, charginos and sleptons may be too heavy to be pair-produced at a $e^{+} e^{-}$machine with $\sqrt{s}=500 \mathrm{GeV}$.

PACS numbers: 11.30.Pb, 12.60.Jv, 14.80.Ly
\end{abstract}

\section{INTRODUCTION}

Supersymmetry (SUSY) is at present the only known framework [1] in which the Higgs sector of the Standard Model (SM), so essential for its internal consistency, is technically natural 2]. Supersymmetry is, however, not an exact symmetry in nature. The precise manner in which SUSY is broken is not known at present. However, the necessary SUSY breaking can be introduced through soft supersymmetry breaking terms that do not reintroduce quadratic divergences in the Higgs mass, and thereby do not disturb the stability of the hierarchy between the weak scale and the large grand unified (GUT) scale. Such terms can typically arise in supergravity theories, in which local supersymmetry is spontaneously broken in a hidden sector, and is then transmitted to the visible sector via gravitational interactions. A particularly attractive implementation of the idea of supersymmetry, with soft supersymmetry breaking terms generated by supergravity, is the Minimal Supersymmetric Standard Model (MSSM) obtained by simply introducing the supersymmetric partners of the of the SM states, and introducing an additional Higgs doublet, with opposite hypercharge to that of the SM Higgs doublet, in order to cancel the gauge anomalies and generate masses for all the fermions of the Standard Model 3, 4]. In order for broken supersymmetry to be effective in protecting the weak scale against large radiative corrections, the supersymmetric partners of the SM particles should have masses of the order of a few hundred $\mathrm{GeV}$. Their discovery is one of the main goals of present and future accelerators. In particular, a $e^{+} e^{-}$ linear collider with a high luminosity $\mathcal{L}=500 \mathrm{fb}^{-1}$, and a center-of-mass energy of $\sqrt{s}=500 \mathrm{GeV}$ in the first stage, will be an important tool in determining the parameters of the low energy supersymmetric model with a high precision [5, 6, 7, 8, 9]. Furthermore, polarisation of the electron (and positron) beam can enhance the capability of such a linear collider [10] in unravelling the structure of the underlying supersymmetric model.

In the minimal supersymmetric standard model the fermionic partners of the two Higgs doublets $\left(H_{1}, H_{2}\right)$ mix with the fermionic partners of the gauge bosons to produce four neutralino states $\tilde{\chi}_{i}^{0}, i=1,2,3,4$, and two chargino states $\tilde{\chi}_{j}^{ \pm}, j=1,2$. In the MSSM with $R$-parity $\left(R_{p}\right)$ conservation, the lightest neutralino state is expected to be the lightest supersymmetric particle (LSP). The neutralino states of the minimal supersymmetric standard model with $R_{p}$ conservation have been studied in great detail, because the lightest neutralino, being the LSP, is the end product of any process involving supersymmetric particle in the final state.

\footnotetext{
* Electronic address: rahul@imsc.res.in

$\dagger$ Electronic address: ppandita@nehu.ac.in

‡lectronic address: sharma@imsc.res.in

$\S$ permanent address
} 
However, the MSSM suffers from the so-called $\mu$ problem associated with the bilinear term connecting the two Higgs doublet superfields $H_{1}$ and $H_{2}$ in the superpotential. An elegant solution to this problem is to postulate the existence of a chiral electroweak gauge singlet superfield $S$, and couple it to the two Higgs doublet superfields $H_{1}$ and $H_{2}$ via a dimensionless trilinear term $\lambda H_{1} H_{2} S$ in the superpotential. When the scalar component of the singlet superfield $S$ obtains a vacuum expectation value, a bilinear term $\lambda H_{1} H_{2}\langle S\rangle$ involving the two Higgs doublets is naturally generated. Furthermore, when this scalar component of the chiral singlet superfield $S$ acquires a vacuum expectation value of the order of the $S U(2)_{L} \times U(1)_{Y}$ breaking scale, it gives rise to an effective value of $\mu\left(\mu_{e f f} \equiv \lambda\langle S\rangle=\lambda x\right)$ of the order of the electroweak scale. However, the inclusion of the singlet superfield leads to additional trilinear superpotential coupling $(\kappa / 3) S^{3}$ in the model, the so called nonminimal, or next-to-minimal [1, 12, 13, 14, 15, 16, 17], supersymmetric standard model (NMSSM). The absence of $H_{1} H_{2}$ term, and the absence of tadpole and mass couplings, $S$ and $S^{2}$ in the NMSSM is made natural by postulating a suitable discrete symmetry [18, 19]. The NMSSM is attractive on account of the simple resolution it offers to the $\mu$ problem, and of the scale invariance of its classical action in the supersymmetric limit. Since no dimensional supersymmetric parameters are present in the superpotential of NMSSM, it is the simplest supersymmetric extension of the Standard Model in which the electroweak scale originates from the supersymmetry breaking scale only. Its enlarged Higgs sector may help in relaxing the fine-tuning and little hierarchy problems of the MSSM [20], thereby opening new perspectives for the Higgs boson searches at high energy colliders [21, 22], and for dark matter searches [23]. In the nonminimal supersymmetric standard model the mixing of fermionic partners of Higgs and gauge bosons [24, 25, 26] produces five neutralino states $\tilde{\chi}_{i}^{0}$, $i=1,2,3,4,5$, and two chargino states $\tilde{\chi}_{j}^{ \pm}, j=1,2$. Furthermore, because of the presence of the fermionic partner of the singlet Higgs boson, the neutralino states can have an admixture of this $S U(2)_{L} \times U(1)_{Y}$ singlet fermion, thereby affecting the phenomenology of the neutralinos in the nonminimal supersymmetric standard model.

The lightest neutralino state $\left(\tilde{\chi}_{1}^{0}\right)$ of MSSM or NMSSM, being typically the LSP, is stable and therefore, a possible dark matter candidate [27, 28]. Since the neutralinos are among the lightest particles in low energy supersymmetric models, they are expected to be the first states to be produced at the colliding beam experiments. At an electron-positron collider, such as the International Linear Collider (ILC), they can be directly produced in pairs

$$
e^{+}+e^{-} \rightarrow \tilde{\chi}_{i}^{0}+\tilde{\chi}_{j}^{0}
$$

which proceeds via $Z$ boson and selectron exchange [29, 30]. In collider experiments the LSP escapes detection such that the direct production of the lightest neutralino pair

$$
e^{+}+e^{-} \rightarrow \tilde{\chi}_{1}^{0}+\tilde{\chi}_{1}^{0}
$$

is invisible. Therefore, one must look for the signature of neutralinos in the radiative process

$$
e^{+}+e^{-} \rightarrow \tilde{\chi}_{1}^{0}+\tilde{\chi}_{1}^{0}+\gamma
$$

where the final photon is radiated off of the incoming beams or the exchanged selectrons. We note that this process is suppressed by the square of the electromagnetic coupling. However, it might be the first proceess where the lightest supersymmetric states could be observed at colliders. The signal of the radiative process (I.3) is a single high energy photon with the missing energy carried away by the neutralinos. The process (I.3) has been studied in detail in the minimal supersymmetric model 31, 32, 33, 34, 35, 36, 37, 38, 39, 40, 41. In these studies, different approximations have been used in calculating the cross section for (I.3), and the focus has been on LEP energies and special neutralino mixing, especially the case where the neutralino is a pure photino [31, 32, 33, 34, 35, 36, 37, 38].

More recently, calculations have been carried out in the context of MSSM assuming general neutralino mixing [39, 40, 41]. Some of these studies underline the importance of longitudinal [39] and even transverse beam polarisations. On the other hand, the signature "photon plus missing energy" has been studied in detail by different LEP collaborations ALEPH [42], DELPHI [43], L3 [44], and OPAL [45, 46]. We recall that in the SM, the radiative neutralino process $e^{+} e^{-} \rightarrow \nu \bar{\nu} \gamma$ is the leading process with this signature, for which the cross section depends on the number $N_{\nu}$ of light neutrino species [47]. This signature has, thus, been used to measure $N_{\nu}$, which has been found to be consistent with three. Furthermore, the LEP collaborations have found no deviations from the SM prediction, and, therefore, only bounds on the masses of supersymmetric particles have been set [42, 43, 44, 46]. This process is also important in determining collider bounds on a very light neutralino [48]. For a review of the experimental situation, see Ref. [49].

Most of the theoretical studies on radiative neutralino production in the literature have been carried out in the framework of the minimal supersymmetric standard model. This includes calculations relevant to ILC with 
a high center-of-mass energy, high luminosity and logitudinally polarized beams, as well as study of the SM background from the radiative neutrino production

$$
e^{+} e^{-} \rightarrow \nu+\bar{\nu}+\gamma
$$

and the MSSM background from radiative sneutrino production

$$
e^{+} e^{-} \rightarrow \tilde{\nu}+\tilde{\nu}^{*}+\gamma
$$

It has been pointed out [50] that the discovery potential of the ILC might be significantly extended if both beams are polarized, especially if other SUSY states like heavier neutralino, chargino or even slepton pairs are too heavy to be produced at the first stage of the ILC at $\sqrt{s}=500 \mathrm{GeV}$.

In this paper we shall consider the radiative process (I.3) in the nonminimal supersymmetric standard model. As discussed above, the nonminimal supersymmetric standard model is an attractive alternative to the MSSM, which solves the $\mu$ problem of the minimal supersymmetric standard model, and in which the weak scale originates from the supersymmetry breaking scale only. Furthermore, the NMSSM has five neutralino states in its spectrum, and there is an admixture of a singlet state in the neutralino states, which may affect the radiative neutralino production process ( $\underline{\text { I.3 }}$. On the other hand, the background processes from the SM and supersymmetry are not affected by the spectrum of neutralino states. We shall compare and contrast the signal for the radiative neutralino process in the NMSSM with that in the MSSM, and study in detail the dependence of the cross sections on the parameters of the neutralino sector.

The plan of the paper is as follows. In Sec. II we discuss the cross section for the signal process (I.3) in NMSSM, and compare and contrast it with the corresponding cross section in the MSSM. Here we recall the basic features of the neutralino mixing matrix in NMSSM and MSSM, and the couplings of the neutralinos relevant for our calculations, as well as the cross section and the phase space for the signal process. Here we also describe the cuts on the photon angle and energy that are used to regularise the infrared and collinear divergences in the tree level cross section. We then describe the typical set of input parameters for the NMSSM that are used in our numerical evaluation of the cross sections. The set of parameters that we use are obtained by imposing various experimental and theoretical constraints on the parameter space of NMSSM. On the other hand, for MSSM we use the typical benchmark parameter set of the Snowmass Points and Slopes 1a ( SPS 1a) scenario [51], except when otherwise indicated. We analyse numerically the dependence of the cross section on the parameters of the neutralino sector, and on the selectron masses. In Sec. III we discuss the backgrounds to the radiative neutralino production process (I.3) from the SM and supersymmetric processes. Here we also define a statistical significance for measuring an excess of photons from radiative neutralino production over the backgrounds. We summarise our results and conclusions in Sec. IV.

\section{RADIATIVE NEUTRALINO PRODUCTION}

\section{A. Neutralino Mass Matrix, Lagrangian and Couplings}

In order to calculate the cross section for the radiative production of neutralinos

$$
e^{-}\left(p_{1}\right)+e^{+}\left(p_{2}\right) \rightarrow \tilde{\chi}_{1}^{0}\left(k_{1}\right)+\tilde{\chi}_{1}^{0}\left(k_{2}\right)+\gamma(q),
$$

where the symbols in the brackets denote the four momenta of the respective particles, we need to compute the couplings of the neutralinos to electrons and the scalar partners of electrons, the selectrons. These can be obtained from the neutralino mixing matrix. To obtain the neutralino mixing matrix for the MSSM, we recall that the neutralino mass matrix obtains contributions from part of the MSSM superpotential

$$
W_{\mathrm{MSSM}}=\mu \mathrm{H}_{1} \mathrm{H}_{2},
$$

where $H_{1}$ and $H_{2}$ are the two Higgs doublet chiral superfields, and $\mu$ is the supersymmetric Higgs(ino) parameter. In addition to the contribution from the superpotential, the neutralino mass matrix receives contributions from the interactions between gauge and matter multiplets, as well as contributions from the soft supersymmetry breaking masses for the gauginos. Including all these contributions, the neutralino mass matrix, in the bino, wino, higgsino basis $\left(-i \lambda^{\prime},-i \lambda^{3}, \psi_{H_{1}}^{1}, \psi_{H_{2}}^{2}\right)$ can be written as [52, 53]

$$
M_{\mathrm{MSSM}}=\left(\begin{array}{cccc}
M_{1} & 0 & -m_{Z} \sin \theta_{w} \cos \beta & m_{Z} \sin \theta_{w} \sin \beta \\
0 & M_{2} & m_{Z} \cos \theta_{w} \cos \beta & -m_{Z} \cos \theta_{w} \sin \beta \\
-m_{Z} \sin \theta_{w} \cos \beta & m_{Z} \cos \theta_{w} \cos \beta & 0 & -\mu \\
m_{Z} \sin \theta_{w} \sin \beta & -m_{Z} \cos \theta_{w} \sin \beta & -\mu & 0
\end{array}\right),
$$


where $M_{1}$ and $M_{2}$ are the $U(1)_{Y}$ and the $S U(2)_{L}$ soft supersymmetry breaking gaugino mass parameters, respectively, and $\tan \beta=v_{2} / v_{1}$ is the ratio of the vacuum expectation values of the neutral components of the two Higgs doublet fields $H_{1}$ and $H_{2}$, respectively. Furthermore, $m_{Z}$ is the $Z$ boson mass, and $\theta_{w}$ is the weak mixing angle. In the $\mathrm{CP}$ conserving case, $M$ is a real symmetric matrix and can be diagonalised by an orthogonal matrix. Since at least one eigenvalue of $M$ is negative, we can use a unitary matrix $N$, the neutralino mixing matrix, to get a positive semidefinite diagonal matrix [52] with the neutralino masses $m_{\chi_{i}^{0}}$ :

$$
N^{*} M_{\mathrm{MSSM}} N^{-1}=\operatorname{diag}\left(m_{\chi_{1}^{0}}, m_{\chi_{2}^{0}}, m_{\chi_{3}^{0}}, m_{\chi_{4}^{0}}\right) .
$$

We note that that the transformation Eq. (II.4) is only a similarity transformation if $N$ is real.

For the NMSSM, the relevant part of the superpotential is

$$
W_{\mathrm{NMSSM}}=\lambda S H_{1} H_{2}-\frac{\kappa}{3} S^{3},
$$

where $S$ is the Higgs singlet chiral superfield. In the basis $\left(-i \lambda^{\prime},-i \lambda^{3}, \psi_{H_{1}}^{1}, \psi_{H_{2}}^{2}, \psi_{S}\right)$, the neutralino mass matrix for the NMSSM can then be written as [24, 25]

$$
M_{\mathrm{NMSSM}}=\left(\begin{array}{ccccc}
M_{1} & 0 & -m_{Z} \sin \theta_{w} \cos \beta & m_{Z} \sin \theta_{w} \sin \beta & 0 \\
0 & M_{2} & m_{Z} \cos \theta_{w} \cos \beta & -m_{Z} \cos \theta_{w} \sin \beta & 0 \\
-m_{Z} \sin \theta_{w} \cos \beta & m_{Z} \cos \theta_{w} \cos \beta & 0 & -\lambda x & -\lambda v_{2} \\
m_{Z} \sin \theta_{w} \sin \beta & -m_{Z} \cos \theta_{w} \sin \beta & -\lambda x & 0 & -\lambda v_{1} \\
0 & 0 & -\lambda v_{2} & -\lambda v_{1} & 2 \kappa x
\end{array}\right),
$$

where $\langle S\rangle=x$ is the vacuum expectation value of the singlet Higgs field. As in the case of MSSM, we can use a unitary matrix $N^{\prime}$ to get a positive semidefinite diagonal matrix with the neutralino masses $m_{\chi_{i}^{0}}[24$, 25]:

$$
N^{\prime *} M_{\mathrm{NMSSM}} N^{\prime-1}=\operatorname{diag}\left(m_{\chi_{1}^{0}}, m_{\chi_{2}^{0}}, m_{\chi_{3}^{0}}, m_{\chi_{4}^{0}} m_{\chi_{5}^{0}}\right) .
$$

The Lagrangian for the interaction of neutralinos, electrons, selectrons and $Z$ bosons for MSSM is given by 52 ]

$$
\begin{aligned}
\mathcal{L}= & \left(-\frac{\sqrt{2} e}{\cos \theta_{w}} N_{11}^{*}\right) \bar{f}_{e} P_{L} \tilde{\chi}_{1}^{0} \tilde{e}_{R}+\frac{e}{\sqrt{2} \sin \theta_{w}}\left(N_{12}+\tan \theta_{w} N_{11}\right) \bar{f}_{e} P_{R} \tilde{\chi}_{1}^{0} \tilde{e}_{L} \\
& +\frac{e}{4 \sin \theta_{w} \cos \theta_{w}}\left(\left|N_{13}\right|^{2}-\left|N_{14}\right|^{2}\right) Z_{\mu} \overline{\tilde{\chi}}_{1}^{0} \gamma^{\mu} \gamma^{5} \tilde{\chi}_{1}^{0} \\
& +e Z_{\mu} \bar{f}_{e} \gamma^{\mu}\left[\frac{1}{\sin \theta_{w} \cos \theta_{w}}\left(\frac{1}{2}-\sin ^{2} \theta_{w}\right) P_{L}-\tan \theta_{w} P_{R}\right] f_{e}+\text { h.c. },
\end{aligned}
$$

with the electron, selectron, neutralino and $Z$ boson fields denoted by $f_{e}, \tilde{e}_{L, R}, \tilde{\chi}_{1}^{0}$, and $Z_{\mu}$, respectively, and $P_{R, L}=\frac{1}{2}\left(1 \pm \gamma^{5}\right)$. The corresponding interaction Lagrangian for NMSSM is obtained from (II.8) by replacing $N_{i j}$ with $N_{i j}^{\prime}$. The different vertices following from (II.8) are shown in Table [.

The couplings of the lightest neutralino to electrons, selectrons and $Z$ boson are determined by the corresponding elements of the neutralino mixing matrix $\left(N_{i j}\right.$ or $\left.N_{i j}^{\prime}\right)$. For numerical calculation of the radiative neutralino cross section in the MSSM, we have chosen to work with the parameters in the SPS 1a scenario [51]. The parameters of the SPS 1a scenario are summarised in Table II However, since in the SPS 1a scenario the value of the parameters $\mu$ and $M_{2}$ are fixed, we shall use a different set of parameters to study the dependence of the neutralino mass and the radiative neutralino production cross section on $\mu$ and $M_{2}$, and on the selectron masses. This set of parameters is shown in Table III We shall call this set of parameters as the MSSM electroweak symmetry breaking scenario (EWSB) [54].

On the other hand, for the NMSSM we use a set of parameters that is obtained by imposing theoretical and experimental constraints on the parameter space of the NMSSM. The parameters that enter the neutralino mass matrix of the NMSSM are, apart from $M_{1}$ and $\left.M_{2}, \tan \beta, \mu(\equiv \lambda<S\rangle=\lambda x\right), \lambda$ and $\kappa$. For $M_{1}, M_{2}$ and $M_{3}$ we use the values which are consistent with the usual GUT relation $M_{1} / \alpha_{1}=M_{2} / \alpha_{2}=M_{3} / \alpha_{3}$. We note that for the MSSM in SPS 1a scenario, the value of the parameter $\tan \beta=10$. In order to remain close to the SPS 1a scenario of MSSM, we have chosen for our numerical calculations in NMSSM $\tan \beta=10$, whereas the rest of the parameters are chosen in such a way that the lightest Higgs boson mass, the lightest neutralino mass and the lightest chargino mass satisfy the present experimental lower limits. We have also imposed on the parameter space of NMSSM the theoretical constraint that there is no charge and color breaking global minimum of the scalar potential, and that a Landau pole does not develop below the grand unified scale $\left(M_{G U T} \approx 10^{16} \mathrm{GeV}\right)$. For instance, for the parameter values $\tan \beta=10, \mu=130 \mathrm{GeV}, \lambda=0.55$ and $\kappa=0.44$, we obtain the lightest 
TABLE I: Vertices corresponding to various terms in the interaction Lagrangian (II.8) for MSSM. In addition we have also shown the vertices for selectron-photon and electron-photon interactions. The vertices for the NMSSM are obtained by replacing $N_{i j}$ with $N_{i j}^{\prime}$.

\begin{tabular}{ll}
\hline Vertex & Vertex Factor \\
\hline right selectron - electron - neutralino & $\frac{-i e \sqrt{2}}{\cos \theta_{w}} N_{11}^{*} P_{L}$ \\
left selectron - electron - neutralino & $\frac{i e}{\sqrt{2} \sin \theta_{w}}\left(N_{12}+\tan \theta_{w} N_{11}\right) P_{R}$ \\
neutralino - $Z^{0}$ - neutralino & $\frac{i e}{4 \sin \theta_{w} \cos \theta_{w}}\left(\left|N_{13}\right|^{2}-\left|N_{14}\right|^{2}\right) \gamma^{\mu} \gamma^{5}$ \\
electron - $Z^{0}$ - electron & $i e \gamma^{\mu}\left[\frac{1}{\sin \theta_{w} \cos \theta_{w}}\left(\frac{1}{2}-\sin ^{2} \theta_{w}\right) P_{L}-\tan \theta_{w} P_{R}\right]$ \\
selectron - photon - selectron & $i e\left(p_{1}+p_{2}\right)^{\mu}$ \\
electron - photon - electron & $i e \gamma^{\mu}$
\end{tabular}

Higgs boson mass of about $123 \mathrm{GeV}$, and the lightest neutralino boson mass of about $77 \mathrm{GeV}$, both of which are phenomenologically acceptable values. As another example, choosing $\tan \beta=10$ and $\mu=225 \mathrm{GeV}$ results in the lightest Higgs boson mass of about $114 \mathrm{GeV}$, the lightest neutralino mass of about $132 \mathrm{GeV}$ but with a charge and color breaking global minimum. Other choices give a Landau Pole below $M_{G U T}$ etc. for some particular set of free parameters. It is important to note that choice of parameters away from tan $\beta=10$ is also possible, for example, $\tan \beta=3, \mu=180 \mathrm{GeV}, \lambda=0.3$ and $\kappa=0.1$ produces a lightest Higgs boson mass of about $114 \mathrm{GeV}$, and the lightest neutralino mass of about $103 \mathrm{GeV}$. We have taken $\tan \beta=10$ for NMSSM simply because we wish to compare our results with MSSM for the typical SPS 1a scenario. The consequence of imposing these constraints on the parameter space of NMSSM, and the resulting masses for various particles for a particular choice of input parameters is summarized in Table IV.

Since the neutralino mass matrix depends on the parameters $\lambda$ and $\kappa$, it is useful to study the possible values of these parameters, with all other parameters fixed, which satisfy the phenomenological and theoretical constraints discussed above. In Fig. 1 we show a plot of $\lambda$ versus $\kappa$, with all other input parameters fixed as in Table IV, and with the lightest neutralino, the lightest Higgs boson, and the lightest chargino mass as in Table IV with a variation of less than 5\%. Fig. 1 shows the range of $\lambda$ and $\kappa$ values that are consistent with all the constraints discussed above for the set of input parameters in Table IV

We note that for the set of input values in Table IV values of $\lambda \lesssim 0.4$, with $\kappa \lesssim 0.22$, lead to an unphysical

TABLE II: Input parameters and resulting masses for various states in the MSSM SPS 1a scenario.

\begin{tabular}{|c|c|c|c|}
\hline $\tan \beta=10$ & $\mu=358 \mathrm{GeV}$ & $M_{2}=192 \mathrm{GeV}$ & $m_{0}=100 \mathrm{GeV}$ \\
\hline \hline$m_{\chi_{1}^{0}}=97 \mathrm{GeV}$ & $m_{\chi_{1}^{ \pm}}=180 \mathrm{GeV}$ & $m_{\tilde{e}_{R}}=136 \mathrm{GeV}$ & $m_{H}=400 \mathrm{GeV}$ \\
\hline$m_{\chi_{2}^{0}}=181 \mathrm{GeV}$ & $m_{\chi_{2}^{ \pm}}=383 \mathrm{GeV}$ & $m_{\tilde{e}_{L}}=196 \mathrm{GeV}$ & $m_{h}=111 \mathrm{GeV}$ \\
\hline
\end{tabular}

TABLE III: Input parameters and resulting masses of various states in MSSM EWSB scenario.

\begin{tabular}{|c|c|c|c|}
\hline $\tan \beta=10$ & $\mu=130 \mathrm{GeV}$ & $M_{1}=150 \mathrm{GeV}$ & $M_{2}=300 \mathrm{GeV}$ \\
\hline$M_{3}=1000 \mathrm{GeV}$ & $A_{t}=3000 \mathrm{GeV}$ & $A_{b}=3000 \mathrm{GeV}$ & $A_{\tau}=1000 \mathrm{GeV}$ \\
\hline \hline$m_{\chi_{1}^{0}}=97 \mathrm{GeV}$ & $m_{\chi_{1}^{ \pm}}=119 \mathrm{GeV}$ & $m_{\tilde{e}_{R}}=143 \mathrm{GeV}$ & $m_{\tilde{\nu}_{e}}=194 \mathrm{GeV}$ \\
\hline$m_{\chi_{2}^{0}}=-141 \mathrm{GeV}$ & $m_{\chi_{2}^{ \pm}}=330 \mathrm{GeV}$ & $m_{\tilde{e}_{L}}=204 \mathrm{GeV}$ & $m_{h}=120 \mathrm{GeV}$ \\
\hline
\end{tabular}


TABLE IV: Input parameters and resuling masses of various states in NMSSM.

\begin{tabular}{|c|c|c|c|}
\hline $\tan \beta=10$ & $\mu=130 \mathrm{GeV}$ & $M_{1}=150 \mathrm{GeV}$ & $M_{2}=300 \mathrm{GeV}$ \\
\hline$M_{3}=1000 \mathrm{GeV}$ & $A_{t}=3000 \mathrm{GeV}$ & $A_{b}=3000 \mathrm{GeV}$ & $A_{\tau}=1000 \mathrm{GeV}$ \\
\hline$\lambda=0.55$ & $\kappa=0.44$ & $A_{\lambda}=880 \mathrm{GeV}$ & $A_{\kappa}=10 \mathrm{GeV}$ \\
\hline \hline$m_{\chi_{1}^{0}}=77 \mathrm{GeV}$ & $m_{\chi_{1}^{ \pm}}=121 \mathrm{GeV}$ & $m_{\tilde{e}_{R}}=149 \mathrm{GeV}$ & $m_{\tilde{\nu}_{e}}=194 \mathrm{GeV}$ \\
\hline$m_{\chi_{2}^{0}}=-158 \mathrm{GeV}$ & $m_{\chi_{2}^{ \pm}}=334 \mathrm{GeV}$ & $m_{\tilde{e}_{L}}=209 \mathrm{GeV}$ & $m_{h}=122 \mathrm{GeV}$ \\
\hline
\end{tabular}

global minimum. On the other hand, values of $\lambda \gtrsim 0.57$, with $\kappa \gtrsim 0.44$, lead to a Landau pole below the GUT scale. Thus, the allowed values of $\lambda$ and $\kappa$, for the given set of input parameters, and for the fixed masses of lightest neutralino, the lightest Higgs boson, and the lightest chargino, as in Table IV lie in a narrow range $0.4 \lesssim \lambda \lesssim 0.57$ for $0.22 \lesssim \kappa \lesssim 0.44$. For definiteness, we have chosen to work with the values of $\lambda=0.55$ and $\kappa=0.44$ in this paper. These values correspond to the peak in the $\lambda$ versus $\kappa$ plot in Fig. 1 .

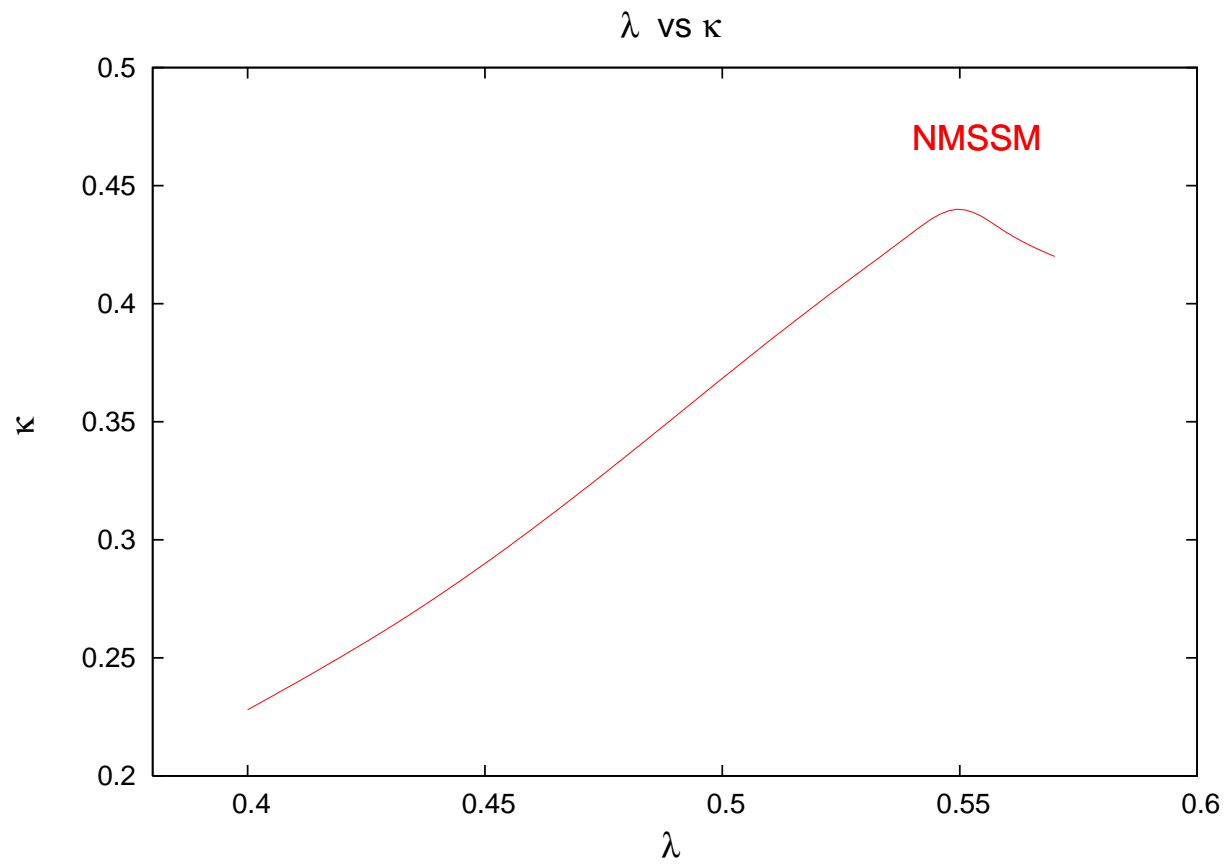

FIG. 1: Plot of $\lambda$ versus $\kappa$ for the set of input parameters in Table IV

For the parameters of Table IV] the composition of the lightest neutralino in NMSSM is given by

$$
N_{1 j}^{\prime}=(0.39,-0.22,0.57,-0.59,0.35)
$$

From the composition (II.9), we see that the lightest neutralino has a sizable singlet component, thereby changing the neutralino phenomenology in the NMSSM as compared to MSSM. For comparison, we also show the particle content of the lightest neutralino in MSSM

$$
N_{1 j}=(0.5,-0.21,0.66,-0.51)
$$

for the parameter set in Table III In Fig. 2 we have plotted the constant contour plots for the mass of lightest neutralino in NMSSM in the $\mu-M_{2}$ plane. We emphasize that the choice of $\mu$ and $M_{2}$ values in this plot have been taken to be consistent with phenomenological and theoretical constraints as described above. For comparison, we have also plotted the corresponding contour plots for MSSM in Fig. 3 with parameters as in Table III. 


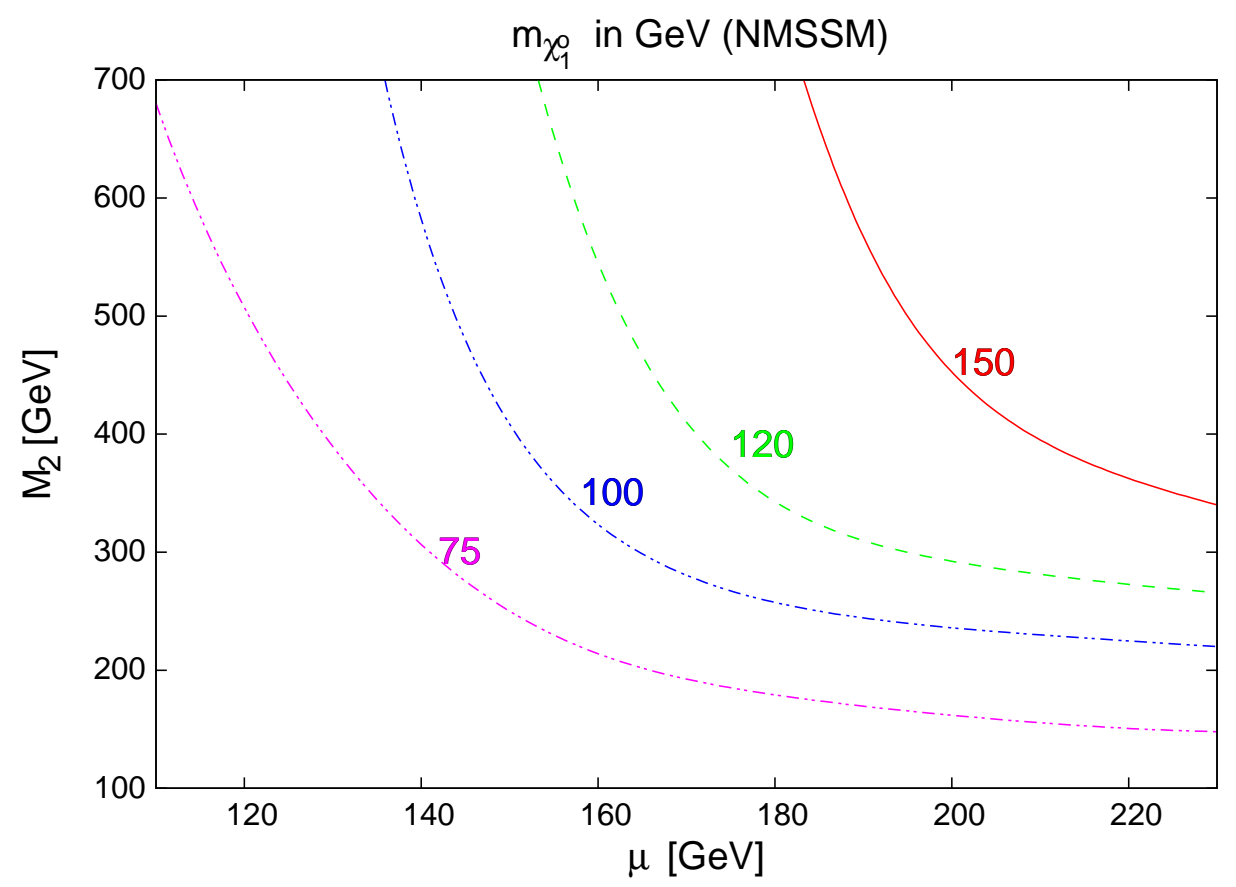

FIG. 2: Contour plots of constant lightest neutralino mass $m_{\chi_{1}^{0}}$ in $\mu-M_{2}$ plane for NMSSM.

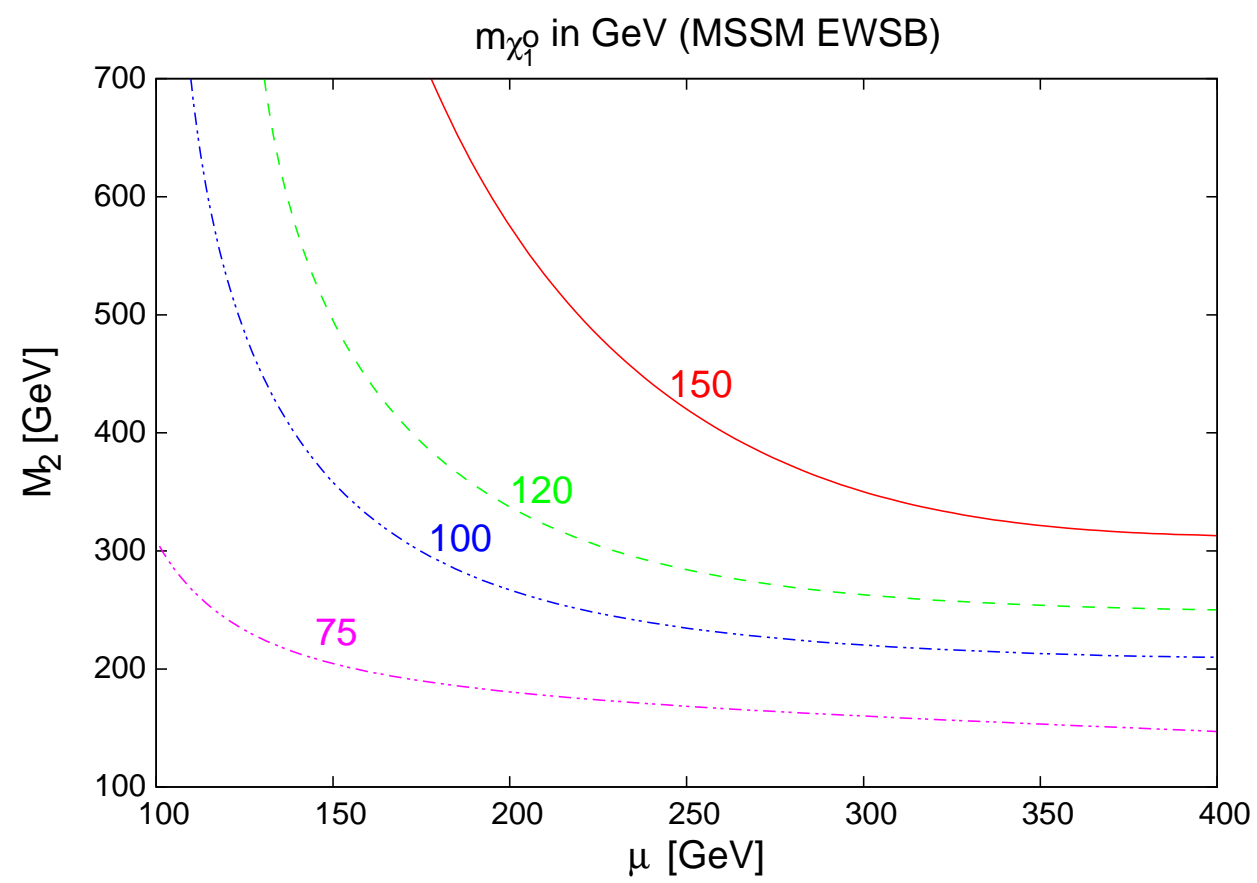

FIG. 3: Contour plots of constant lightest neutralino mass $m_{\chi_{1}^{0}}$ in $\mu-M_{2}$ plane for MSSM.

\section{B. Cross Section for the Signal Process}

In NMSSM, and in MSSM, the process (II.1) proceeds at the tree level via $t$ - and $u$-channel exchange of right and left selectrons $\tilde{e}_{R, L}$, and via $Z$ boson exchange in the $s$ channel. The corresponding Feynman diagrams are shown in Fig. 4. The differential cross section for (II.1) can be written as [33, 55]

$$
d \sigma=\frac{1}{2} \frac{(2 \pi)^{4}}{2 s} \prod_{f} \frac{d^{3} \mathbf{p}_{f}}{(2 \pi)^{3} 2 E_{f}} \delta^{(4)}\left(p_{1}+p_{2}-k_{1}-k_{2}-q\right)|\mathcal{M}|^{2},
$$


where $\mathbf{p}_{f}$ and $E_{f}$ denote the final three-momenta $\mathbf{k}_{1}, \mathbf{k}_{2}, \mathbf{q}$ and the final energies $E_{\chi_{1}}, E_{\chi_{2}}$, and $E_{\gamma}$ of the neutralinos and the photon, respectively. The squared matrix element $|\mathcal{M}|^{2}$ in (II.11) can be written as [33]

$$
|\mathcal{M}|^{2}=\sum_{i \leq j} T_{i j}
$$

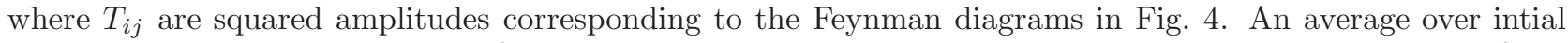
spins and a sum over the spins of the outgoing neutralinos, as well as a sum over the polarizations of the outgoing photon is included in $T_{i j}$. The phase space in (II.11) is described in [33].
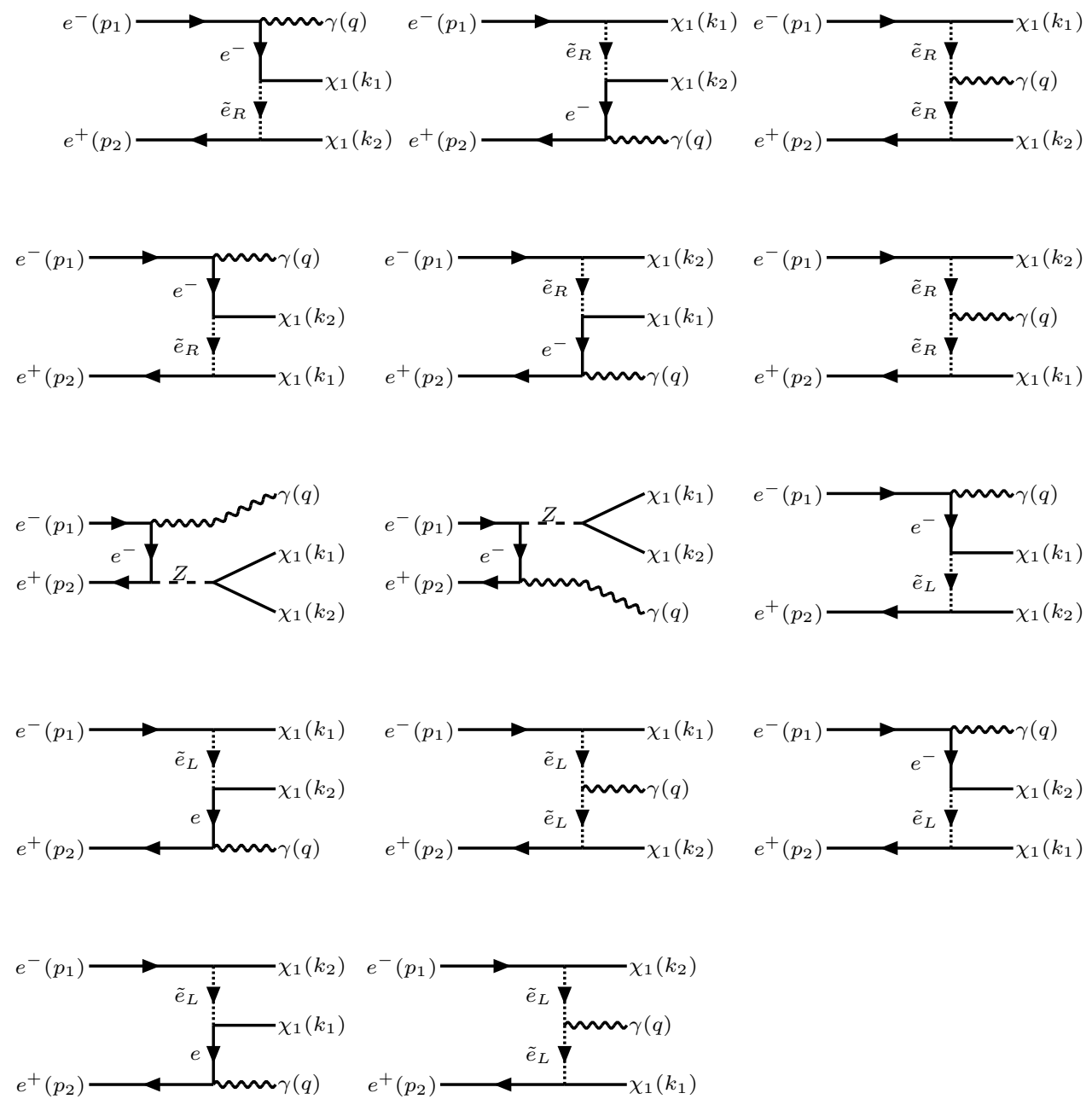

FIG. 4: Feynman diagrams contributing to the radiative neutralino production $e^{+} e^{-} \rightarrow \tilde{\chi}_{1}^{0} \tilde{\chi}_{1}^{0} \gamma$.

\section{Numerical Results}

The tree-level cross section for radiative neutralino production (II.1), and the background from radiative neutrino and sneutrino production, (I.4) and (I.5), have been calculated using the program CalcHEP [54]. The tree level cross sections have infrared and collinear divergences, which need to be regularized. To do this we define the fraction of the beam energy carried by the photon as $x=E_{\gamma} / E_{\text {beam }}$, where $\sqrt{s}=2 E_{\text {beam }}$ is the center of mass energy, and $E_{\gamma}$ is the energy carried away by the photon. We then impose the following cuts on $x$, and on the scattering angle $\theta_{\gamma}$ of the photon [33, 50]:

$$
\begin{aligned}
& 0.02 \leq x \leq 1-\frac{m_{\chi_{1}^{0}}^{2}}{E_{\text {beam }}^{2}}, \\
& -0.99 \leq \cos \theta_{\gamma} \leq 0.99 .
\end{aligned}
$$




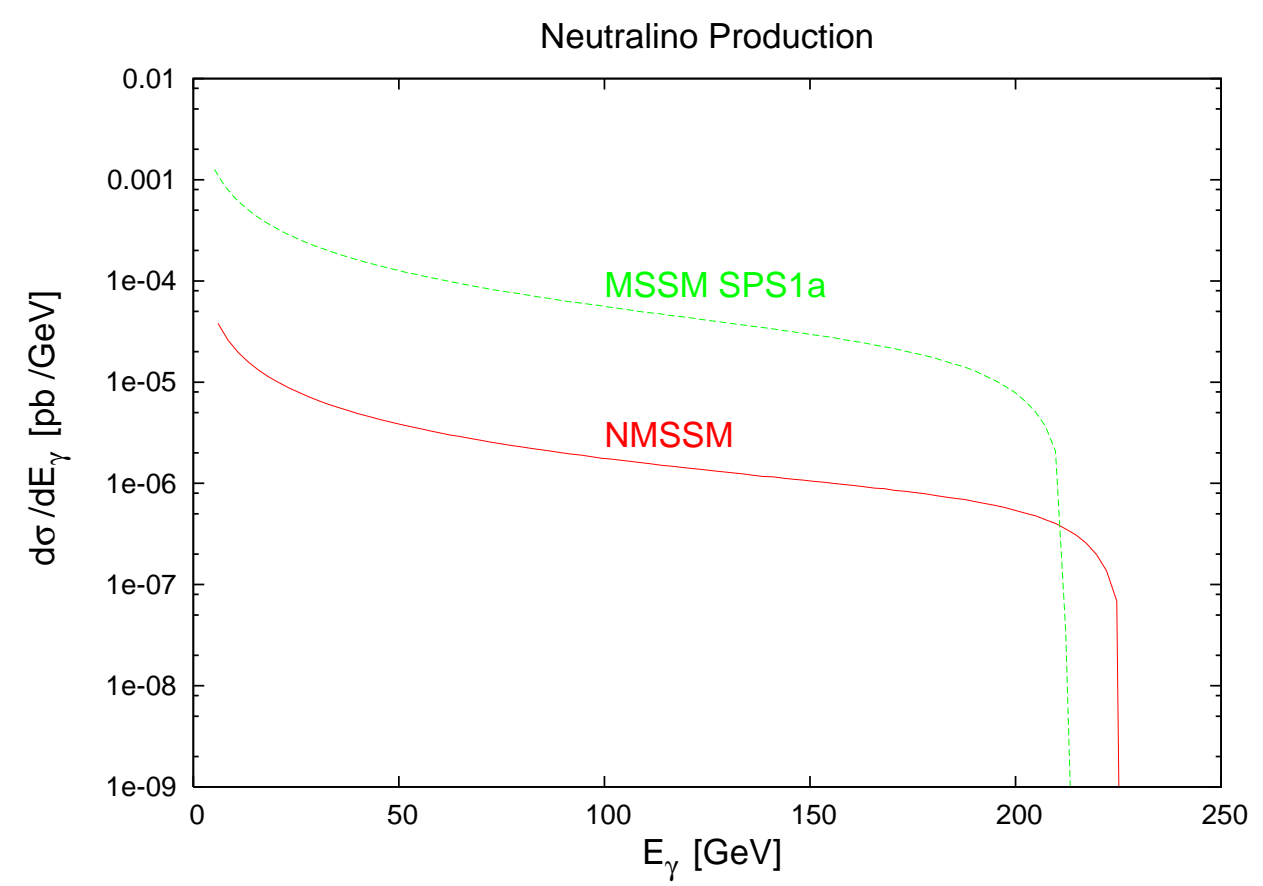

FIG. 5: Photon energy distribution $\frac{d \sigma}{d E_{\gamma}}$ for the radiative neutralino production for NMSSM (red solid line) and for MSSM (green dashed line) at $\sqrt{s}=500 \mathrm{GeV}$.

The lower cut on $x$ in (II.13) corresponds to a photon energy $E_{\gamma}=5 \mathrm{GeV}$ for $\sqrt{s}=500 \mathrm{GeV}$. The upper cut of $\left(1-m_{\chi_{1}^{0}}^{2} / E_{\text {beam }}^{2}\right)$ on $x$ corresponds to the kinematical limit of radiative neutralino production.

In order to implement the cuts on the photon energy in the calculation of the cross sections, we have taken the mass of the lightest neutralino in NMSSM to be $m_{\chi_{1}^{0}}=77 \mathrm{GeV}$ for the parameter set shown in Table IV For MSSM, we take $m_{\chi_{1}^{0}}=97 \mathrm{GeV}$ from the SPS1a scenario. Using Eq. (II.13) we get a fixed upper limit $E_{\gamma}^{\max }=226 \mathrm{GeV}$ for NMSSM and $E_{\gamma}^{\max }=212 \mathrm{GeV}$ for MSSM at $\sqrt{s}=500 \mathrm{GeV}$ for the photon energy. We have used exclusively these cuts for both signal and background processes. Two different mechanisms have been chosen for the production of neutrinos in the background process (1.4), one "with upper cut" and another "without upper cut," for obvious reasons. We note that at $\sqrt{s}=500 \mathrm{GeV}$ and for $m_{\chi_{1}^{0}} \gtrsim 70 \mathrm{GeV}$, this cut reduces a substantial amount of the on-shell $Z$ boson contribution to radiative neutrino production process.

\section{Photon Energy $\left(E_{\gamma}\right)$ Distribution and Total Beam Energy $(\sqrt{s})$ Dependence}

Using the procedure described above, we have calculated the energy distribution of the photons from radiative

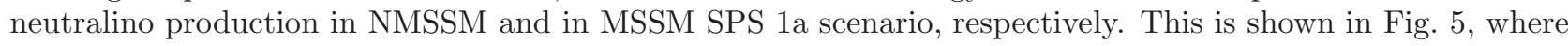
we compare the energy distribution of the photons in the two models. We note that the mass of the lightest neutralino is larger in MSSM SPS1a scenario than in NMSSM for the set of parameters in Tables II and IV respectively, and consequently the upper cut for MSSM is lower than that for NMSSM. We also show the total beam energy $\sqrt{s}$ dependence of the cross sections in Fig. 6 for NMSSM and MSSM SPS 1a, respectively. Due to a smaller value of the mass of neutralino in NMSSM compared to MSSM SPS1 a, the total cross section in NMSSM is less than that in MSSM SPS 1a. From the kinematical endpoint $E_{\gamma}^{\max }=E_{\gamma}^{\max }\left(m_{\chi_{1}^{0}}\right)$ of the energy distribution of the photon from radiative neutralino production, the neutralino mass can, in principle, be determined for $\sqrt{s}=500 \mathrm{GeV}$. We note that although the shape of the energy distribution and the total cross section are similar in NMSS and MSSM, the numerical values for NMSSM are considerably smaller as compared to the MSSM. This is primarily due to the fact that the lightest neutralino in NMSSM has a significant singlet component, thereby reducing the cross section. 


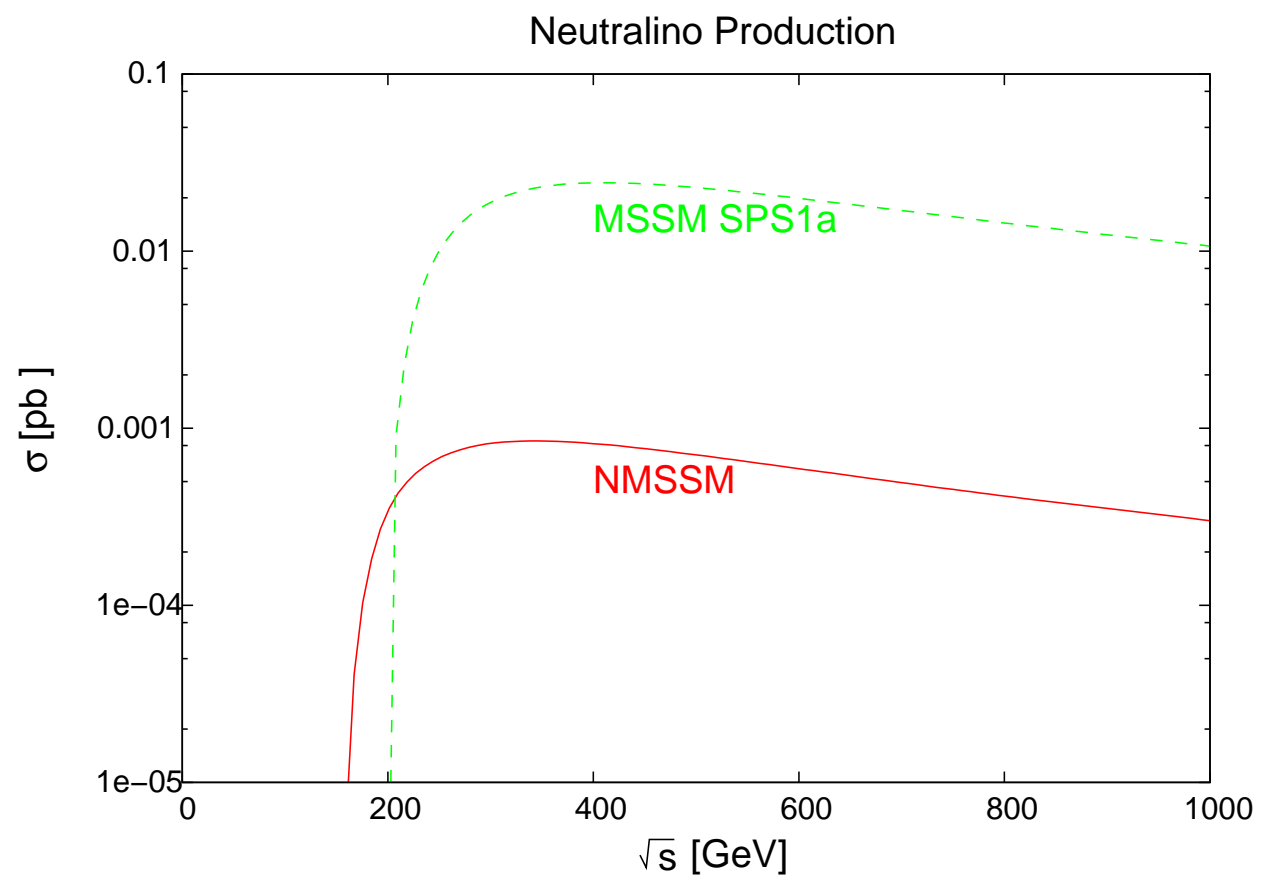

FIG. 6: Total energy $\sqrt{s}$ dependence of the cross sections $\sigma$ for radiative neutralino production $e^{+} e^{-} \rightarrow \tilde{\chi}_{1}^{0} \tilde{\chi}_{1}^{0} \gamma$ for NMSSM (red solid line) and for MSSM SPS1a scenario (green dashed line).

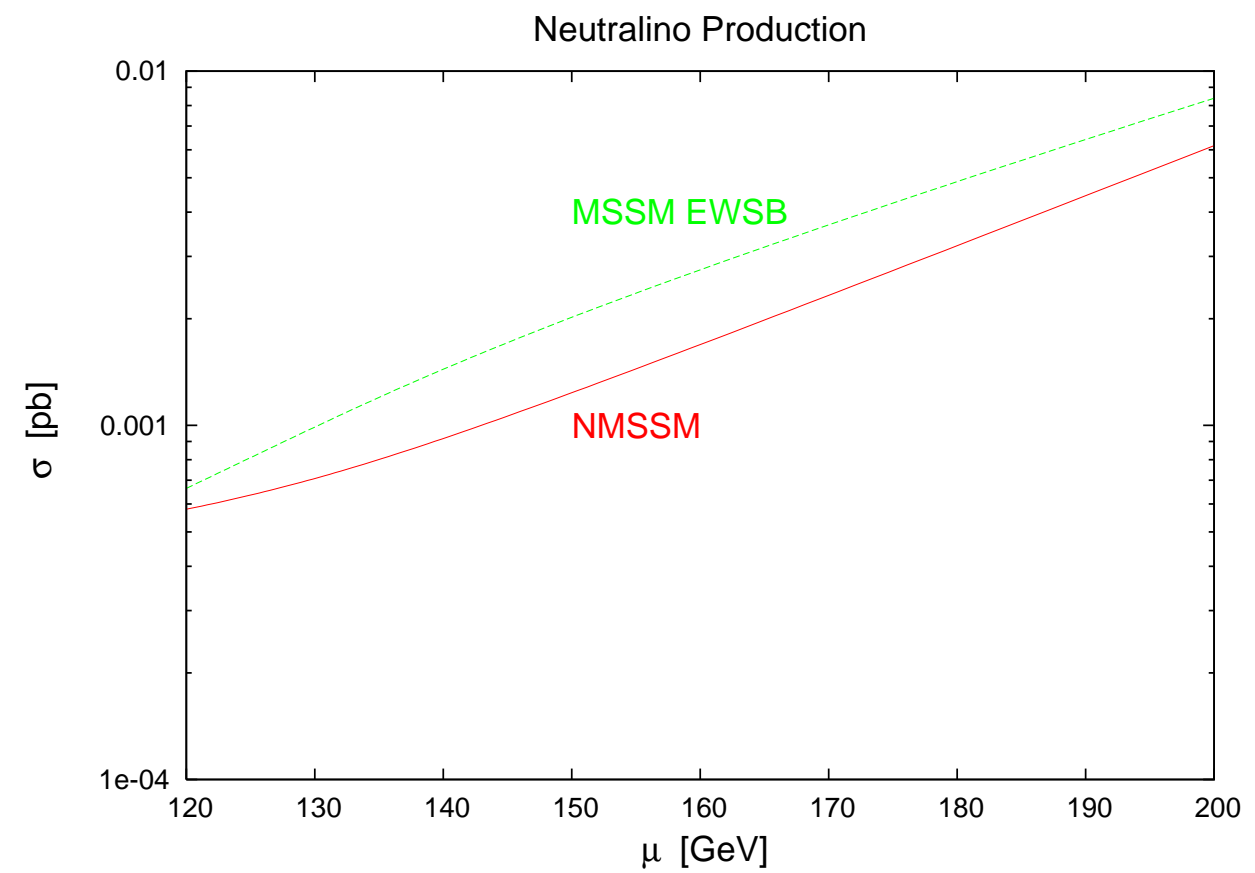

FIG. 7: Total cross-section $\sigma$ for the radiative neutralino production versus $\mu$ for NMSSM (red solid line) and for MSSM in the EWSB scenario (green dashed) at $\sqrt{s}=500 \mathrm{GeV}$. For NMSSM $\mu \equiv \lambda\langle S\rangle=\lambda x$.

\section{Dependence on $\mu$ and $M_{2}$}

Since the neutralino mass matrix, and hence the lightest neutralino mass, depends on $\mu$ and $M_{2}$, it is important to study the dependence of the radiative neutralino cross section on these parameters. In the nonminimal supersymmetric standard model, $\mu(\equiv \lambda<S>=\lambda x)$ and $M_{2}$ are independent parameters. We have, therefore, studied the cross section $\sigma\left(e^{+} e^{-} \rightarrow \tilde{\chi}_{1}^{0} \tilde{\chi}_{1}^{0} \gamma\right)$ as a function of $\mu$ and $M_{2}$ independently. In Fig. 7 


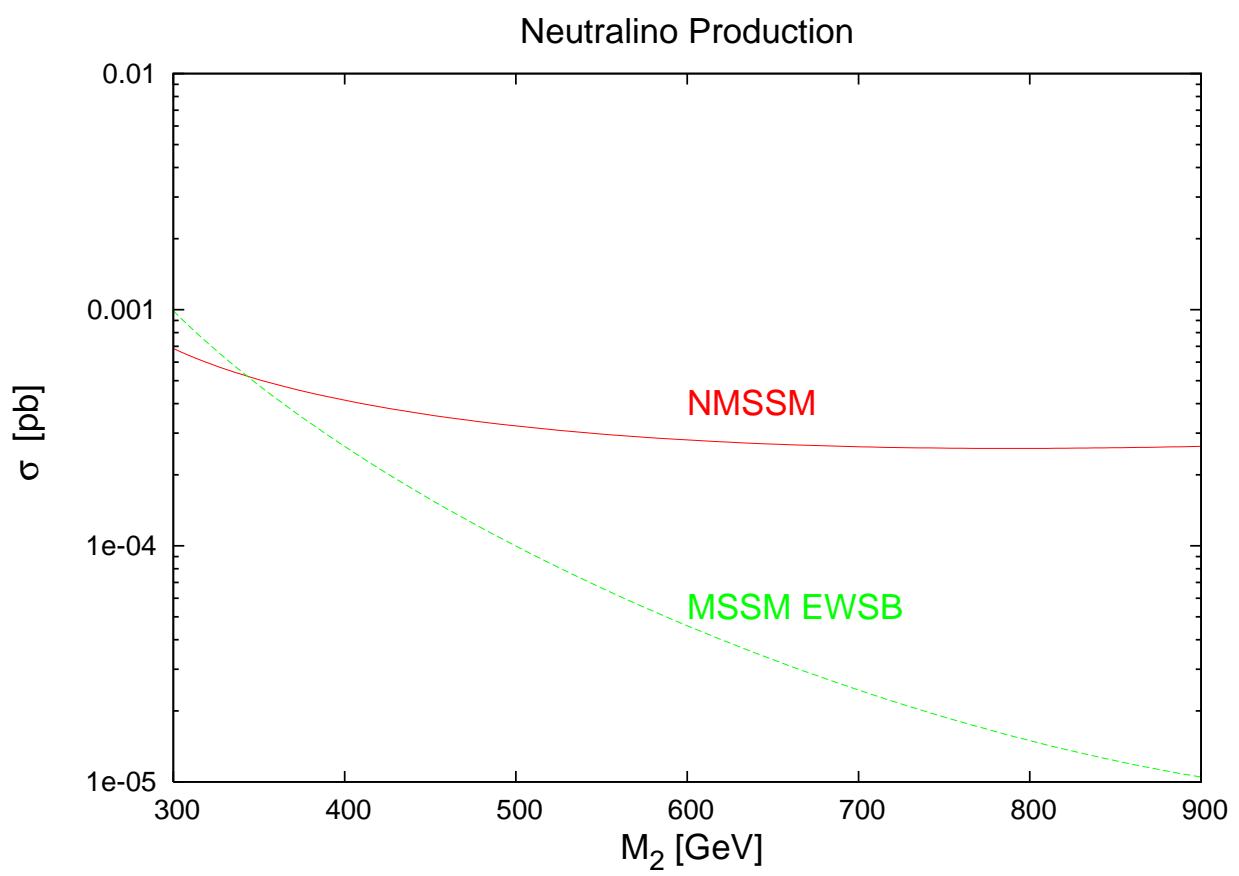

FIG. 8: Total cross section $\sigma$ for the radiative neutralino production versus $M_{2}$ for NMSSM (red solid line) and for MSSM in the EWSB scenario (green dashed) at $\sqrt{s}=500 \mathrm{GeV}$.

we show the $\mu$ dependence of the total cross section for the radiative production of neutralinos for NMSSM as well as MSSM (EWSB). We recall that in the MSSM SPS 1a scenario these parameters are fixed. As is seen from Fig. 7, the total cross section increases with $\mu$. The plot of total cross-section versus $\mu$ in Fig. 7 is plotted in the range $\mu \in[120,200] \mathrm{GeV}$ in NMSSM and in MSSM (EWSB). Note that the parameter values are chosen so as to avoid color and charge breaking minima, absence of Landau pole, and the phenomenological constraints on different particle masses. Furthermore, in Fig. 8 we show the $M_{2}$ dependence of the total cross section for radiative neutralino production for NMSSM and MSSM. The total cross-section decreases with increasing value of $M_{2}$. The graph of total cross-section versus $M_{2}$ in Fig. 8 is plotted for the interval $M_{2} \in[300,900] \mathrm{GeV}$ in NMSSM and in MSSM (EWSB) so as to satisfy the theoretical and phenomenoloigcal constraints described above. It is clear from Fig. 8 that the radiative neutralino production cross section in MSSM decreases sharply as compared to that in NMSSM.

\section{Dependence on the Selectron Masses}

The cross section for radiative neutralino production $\sigma\left(e^{+} e^{-} \rightarrow \tilde{\chi}_{1}^{0} \tilde{\chi}_{1}^{0} \gamma\right)$ proceeds mainly via right and left selectron $\tilde{e}_{R, L}$ exchange in the $t$ and $u$-channels. In the NMSSM and MSSM (EWSB), the selectron masses are independent parameters. In Fig. 9 and Fig. 10 we show the dependence of total cross section of radiative neutralino production on the left and right selectron masses. The cross section is not very sensitive to the selectron masses for both models. Furthermore, the total neutralino production cross section is smaller in NMSSM as compared to MSSM (EWSB) as a function of left as well as right selectron masses.

\section{Photon Energy $\left(E_{\gamma}\right)$ Distribution for the Radiative Production of the Second Lightest Neutralino}

As discussed above, the cross section for the production of the lightest neutralino in NMSSM is rather small compared with the corresponding cross section for the lightest neutralino in the MSSM. It may, therefore, be useful to consider the radiative production of the second lightest neutralino in the NMSSM. For the parameter set of Table 1 the composition of the second lightest neutralino in NMSSM is given by

$$
N_{2 j}^{\prime}=(-0.085,0.1,0.69,0.68,0.19) .
$$




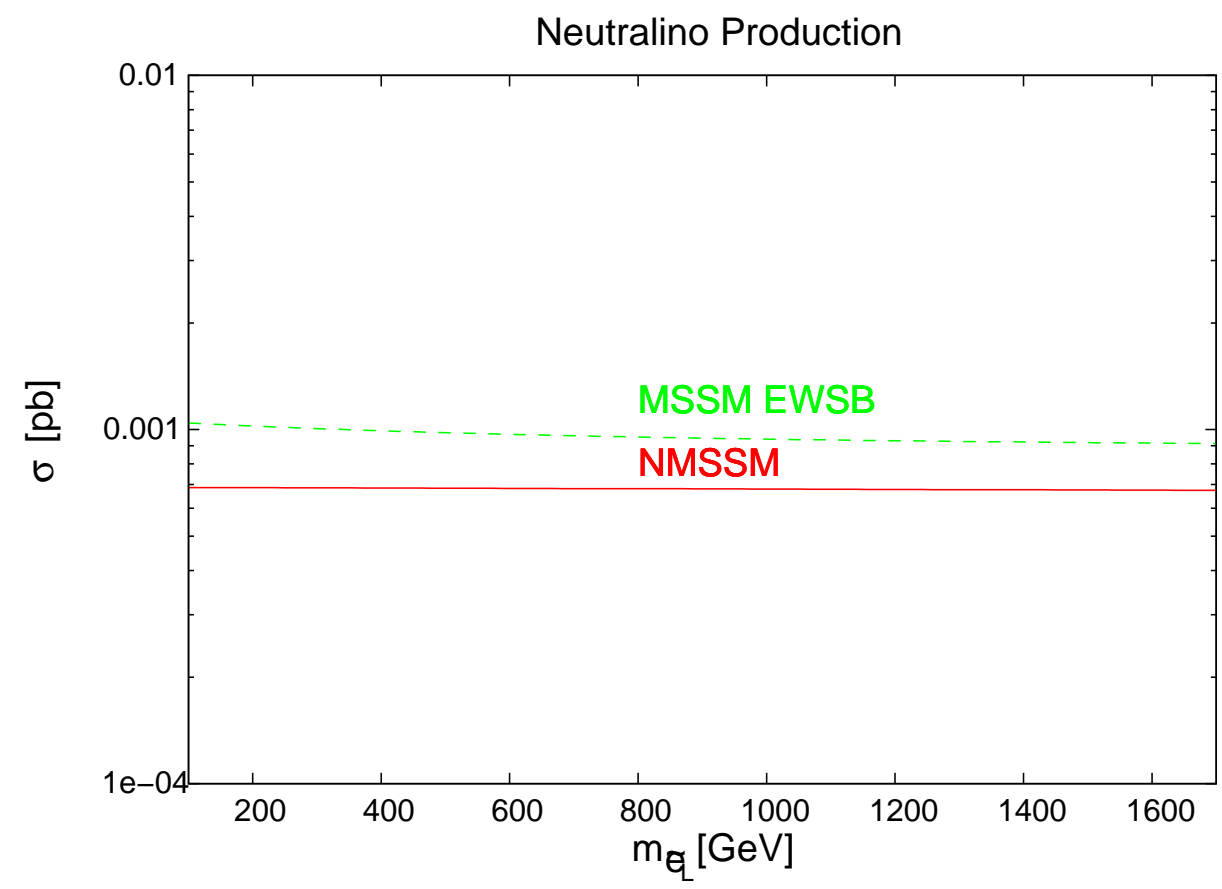

FIG. 9: Total cross section $\sigma$ for the radiative neutralino production versus $m_{\tilde{e}_{L}}$ for NMSSM (red solid line) and for MSSM in the EWSB scenario (green dashed) at $\sqrt{s}=500 \mathrm{GeV}$.

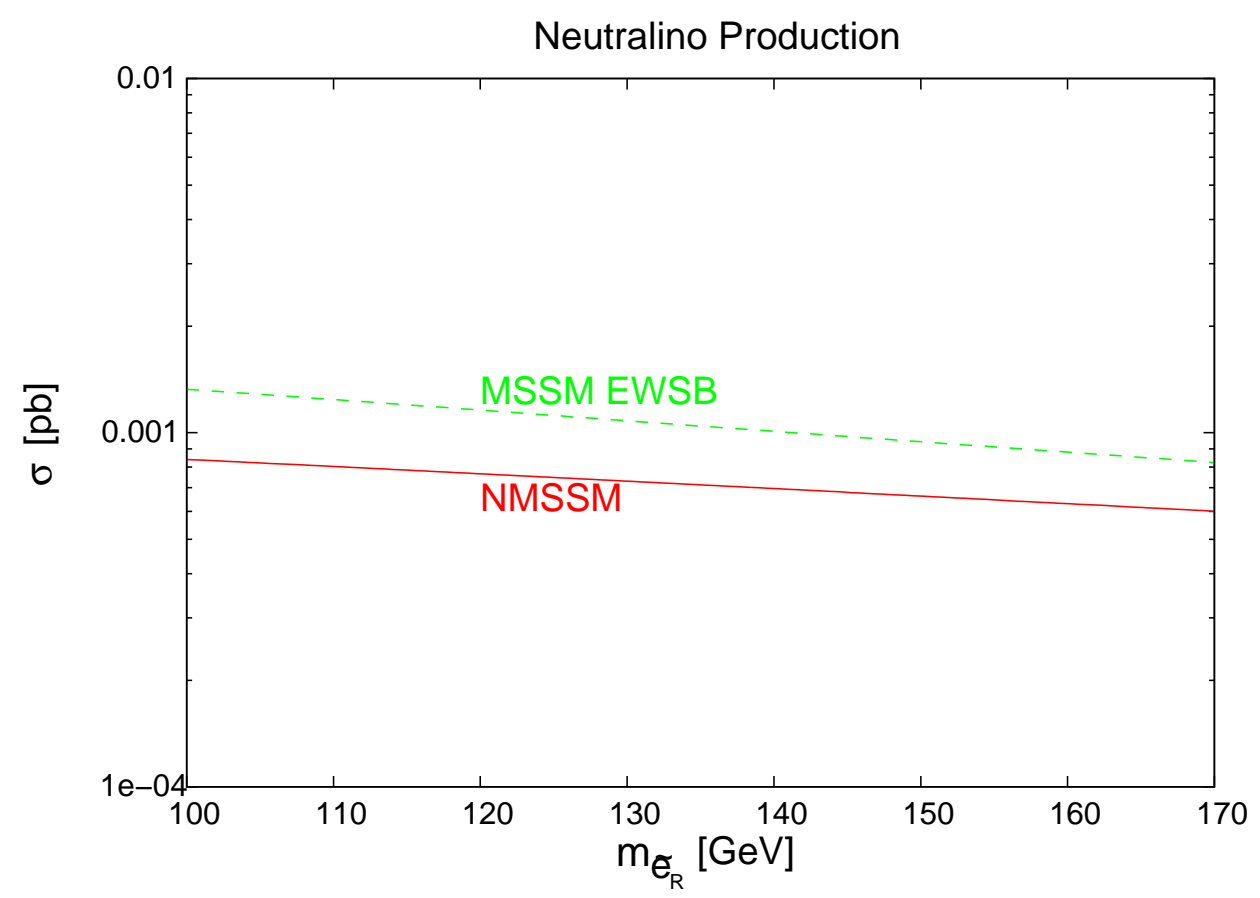

FIG. 10: Total cross section $\sigma$ for the radiative neutralino production versus $m_{\tilde{e}_{R}}$ for NMSSM (red solid line) and for MSSM in the EWSB scenario (green dashed) at $\sqrt{s}=500 \mathrm{GeV}$.

We have calculated the photon energy distribution for the radiative production of the second lightest neutralino in NMSSM for the set of parameters shown in Table IV] This is shown in Fig. 11. For comparison we have also shown the photon energy distribution for the radiative production of the lightest neutralino in MSSM for the SPS 1a scenario. We see that the cross section for the production of the second lightest neutralino in NMSSM is much smaller than the cross section for the lightest neutralino in MSSM. 


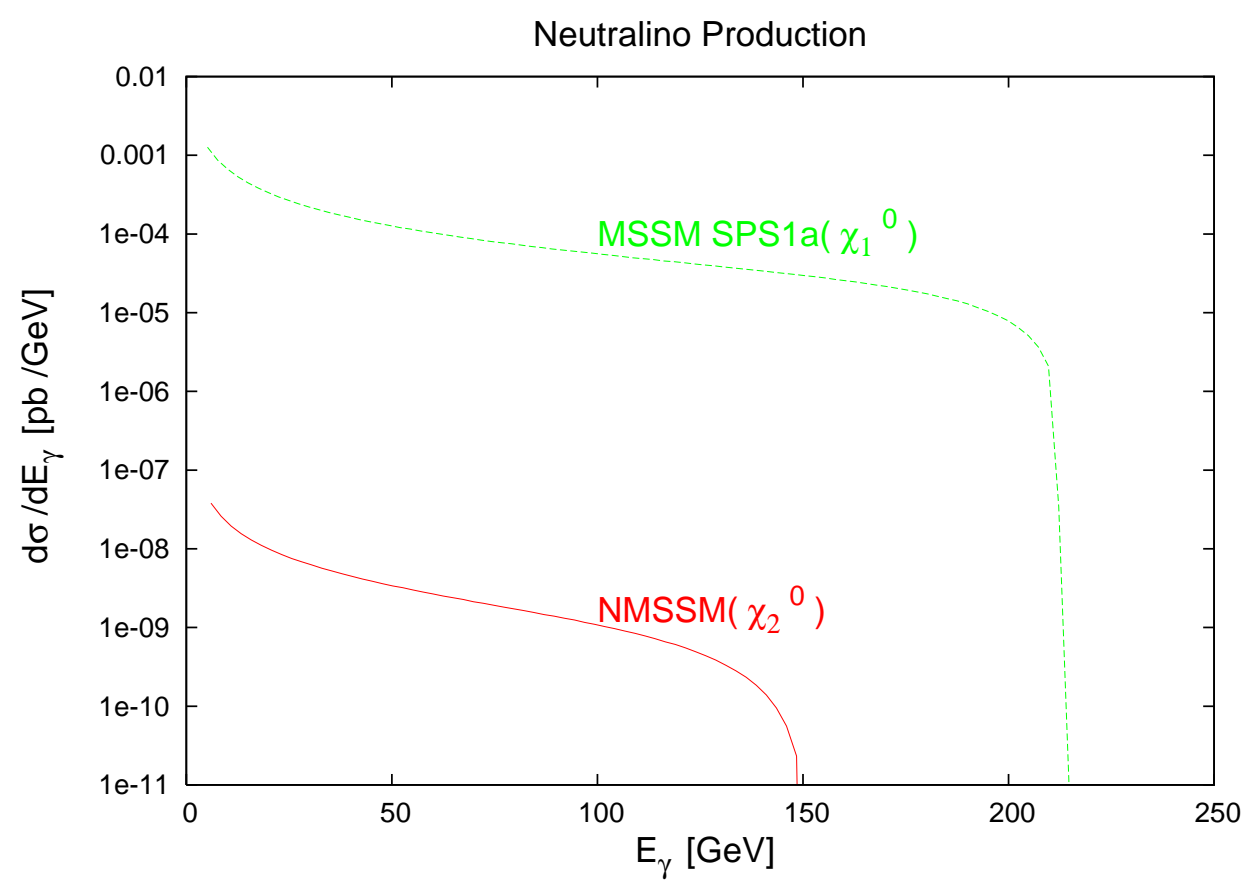

FIG. 11: Photon energy distribution $\frac{d \sigma}{d E_{\gamma}}$ for the radiative production of second lightest neutralino $\left(\chi_{2}^{0}\right)$ for NMSSM $($ red solid line) and for the lightest neutralino $\left(\chi_{1}^{0}\right)$ for MSSM (green dashed line) at $\sqrt{s}=500 \mathrm{GeV}$.

\section{BACKGROUND PROCESSES}

\section{A. The Neutrino Background}

The major background to the radiative neutralino production (II.1) comes from the SM radiative neutrino production process [40, 47, 56, 57, 58]

$$
e^{+}+e^{-} \rightarrow \nu_{\ell}+\bar{\nu}_{\ell}+\gamma, \quad \ell=e, \mu, \tau .
$$

In this process $\nu_{e}$ are produced via $t$-channel $W$ boson exchange, and $\nu_{e, \mu, \tau}$ via $s$-channel $Z$ boson exchange. We show the corresponding Feynman diagrams in Fig. 12. The background photon energy distribution $\frac{d \sigma}{d E_{\gamma}}$ and $\sqrt{s}$ dependence of the cross section $\sigma$ for radiative neutrino production $e^{+} e^{-} \rightarrow \nu \bar{\nu} \gamma$ is the same for both NMSSM and MSSM. As shown in Fig. 13 the photon energy distribution from the radiative neutrino production peaks at $E_{\gamma}=\left(s-m_{Z}^{2}\right) /(2 \sqrt{s}) \approx 242 \mathrm{GeV}$ because of the radiative $Z$ production $\left(\sqrt{s}>m_{Z}\right)$. This photon background from radiative neutrino production can be reduced by imposing an upper cut on the photon energy $x^{\max }=E_{\gamma}^{\max } / E_{\text {beam }}=1-m_{\chi_{1}^{0}}^{2} / E_{\text {beam }}^{2}$, see Eq. (II.13), which is the kinematical endpoint $E_{\gamma}^{\max } \approx 226 \mathrm{GeV}$ of the energy distribution of the photon from radiative neutralino production

$$
m_{\chi_{1}^{0}}^{2}=\frac{1}{4}\left(s-2 \sqrt{s} E_{\gamma}^{\max }\right) .
$$

In order to achieve this, one would have to separate the signal and background processes. This would be possible if the neutralino is heavy enough, such that the endpoint is removed from the $Z^{0}$ peak of the background distribution.

In Fig. 14 we show the $\sqrt{s}$ dependence of the total radiative neutrino cross section. Without the upper cut on the photon energy $x^{\max }$, the background cross section from radiative neutrino production $e^{+} e^{-} \rightarrow \nu \bar{\nu} \gamma$ (green points in Fig. 14 is much larger than the corresponding cross section with the cut (solid red line). However when we impose the cut, the signal cross section from radiative neutralino production is only about one order of magnitude smaller than the background in the case of MSSM, but is nearly three orders of magnitude smaller than the background in the case of NMSSM. 

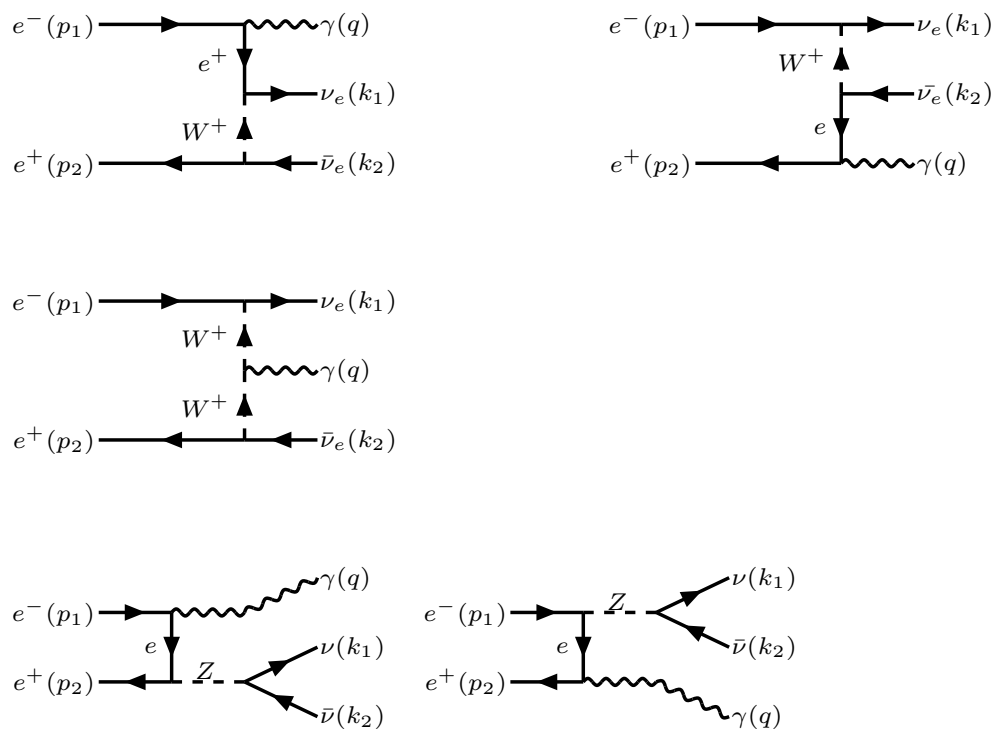

FIG. 12: Feynman diagrams contributing to the radiative neutrino process $e^{+} e^{-} \rightarrow \nu \bar{\nu} \gamma$.

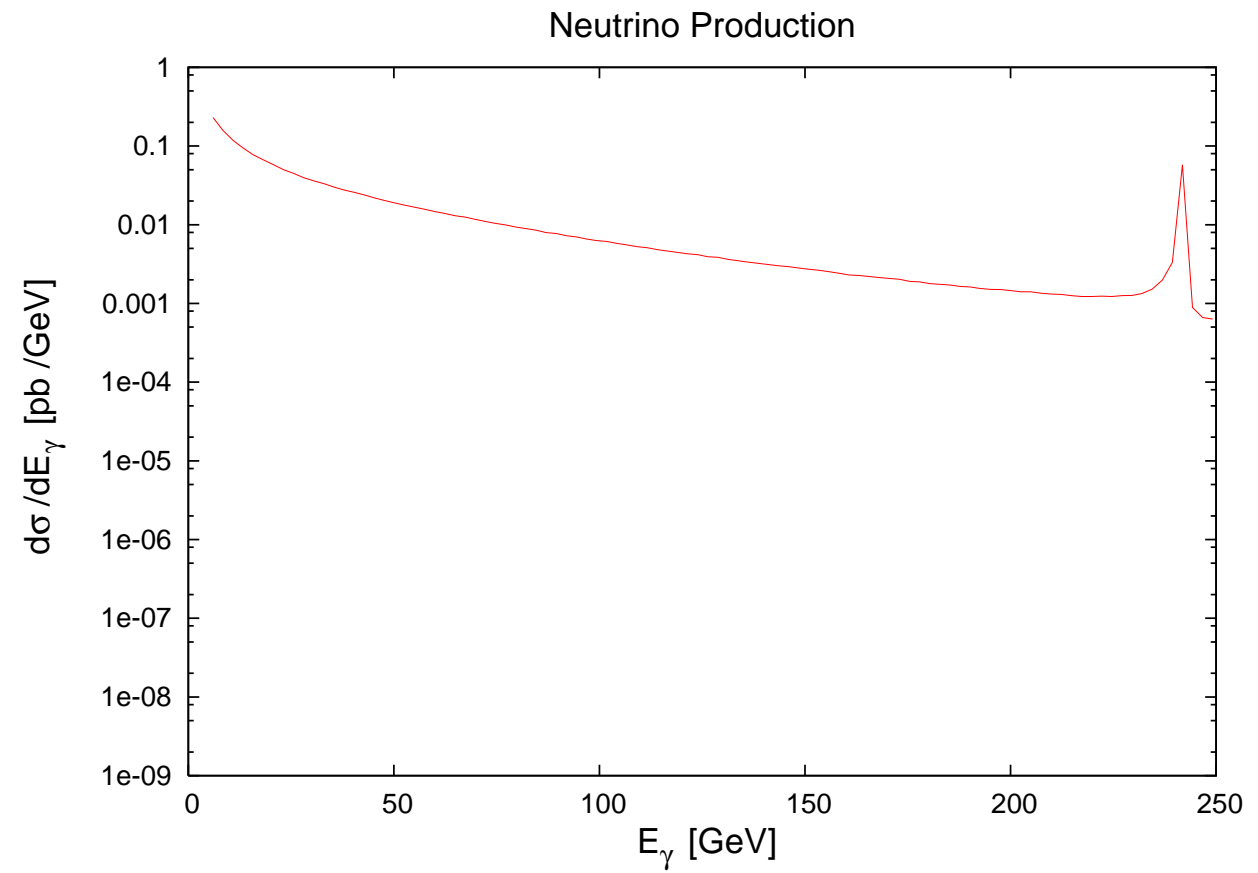

FIG. 13: The photon energy distribution $\frac{d \sigma}{d E_{\gamma}}$ for the radiative neutrino process $e^{+} e^{-} \rightarrow \nu \bar{\nu} \gamma$ at $\sqrt{s}=500 \mathrm{GeV}$.

\section{B. The Supersymmetric Background}

Apart from the SM background coming from (III.1), the radiative neutralino production (II.1) has a background coming from the supersymmetric sneutrino production process [40, 59]

$$
e^{+}+e^{-} \rightarrow \tilde{\nu}_{\ell}+\tilde{\nu}_{\ell}^{*}+\gamma, \quad \ell=e, \mu, \tau .
$$

The lowest order Feynman diagrams contributing to the process (III.3) are shown in Fig. 15. This process receives $t$-channel contributions via virtual charginos for $\tilde{\nu}_{e} \tilde{\nu}_{e}^{*}$-production, as well as $s$-channel contributions from $Z$ boson exchange for $\tilde{\nu}_{e, \mu, \tau} \tilde{\nu}_{e, \mu, \tau}^{*}$-production. In Fig. 16] we show the photon energy distribution $\frac{d \sigma}{d E_{\gamma}}$ for radiative sneutrino production $e^{+} e^{-} \rightarrow \tilde{\nu} \tilde{\nu}^{*} \gamma$ at $\sqrt{s}=500 \mathrm{GeV}$. The total cross section for the radiative sneutrino production is shown in Fig. 17. 


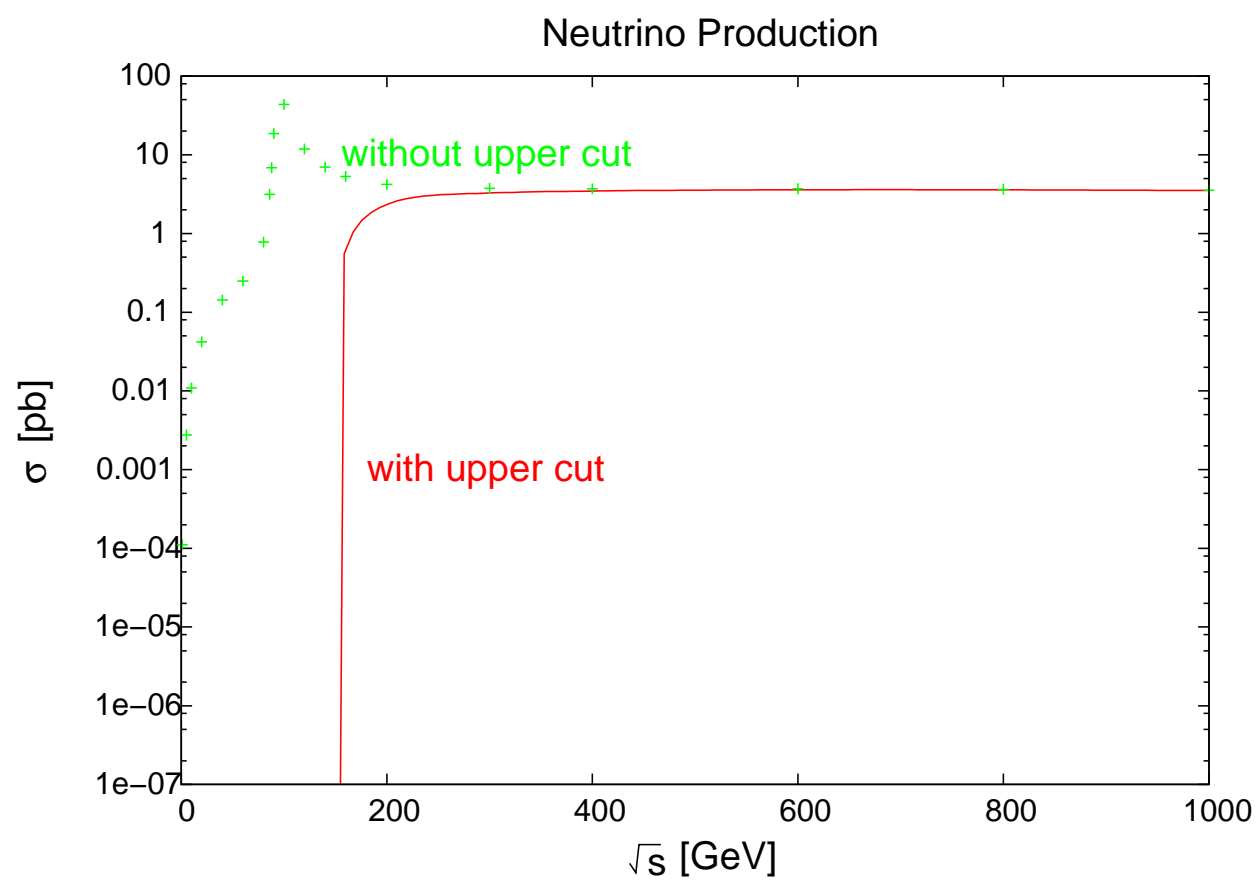

FIG. 14: The total energy $\sqrt{s}$ dependence of the radiative neutrino cross section $\sigma\left(e^{+} e^{-} \rightarrow \nu \bar{\nu} \gamma\right)$ with upper cut (red line) on the photon energy $E_{\gamma}$, and of the radiative neutrino cross section without upper cut (green points) on the photon energy $E_{\gamma}$, see Eq. (II.13).

Radiative sneutrino production (III.3) can be a major supersymmetric background to neutralino production (II.1) if sneutrinos decay mainly invisibly, e.g. via $\tilde{\nu} \rightarrow \tilde{\chi}_{1}^{0} \nu$. This leads to so called "virtual LSP" scenario [40]. However, if kinematically allowed, other visible decay channels like $\tilde{\nu} \rightarrow \tilde{\chi}_{1}^{ \pm} \ell^{\mp}$ reduce the background rate from radiative sneutrino production. For example in the SPS 1a scenario [51, 60] of the MSSM we have $\operatorname{BR}\left(\tilde{\nu}_{e} \rightarrow \tilde{\chi}_{1}^{0} \nu_{e}\right)=85 \%$.

Furthermore, neutralino production $e^{+} e^{-} \rightarrow \tilde{\chi}_{1}^{0} \tilde{\chi}_{2}^{0}$ followed by subsequent radiative neutralino decay 61] $\tilde{\chi}_{2}^{0} \rightarrow \tilde{\chi}_{1}^{0} \gamma$ is also a potential background. However, significant branching ratios $\operatorname{BR}\left(\tilde{\chi}_{2}^{0} \rightarrow \tilde{\chi}_{1}^{0} \gamma\right)>10 \%$ are only obtained for small values of $\tan \beta<5$ and/or $M_{1} \sim M_{2}$ [41, 62, 63]. Thus, we neglect this background, detailed discussions of which can be found in Refs. [62, 63, 64].
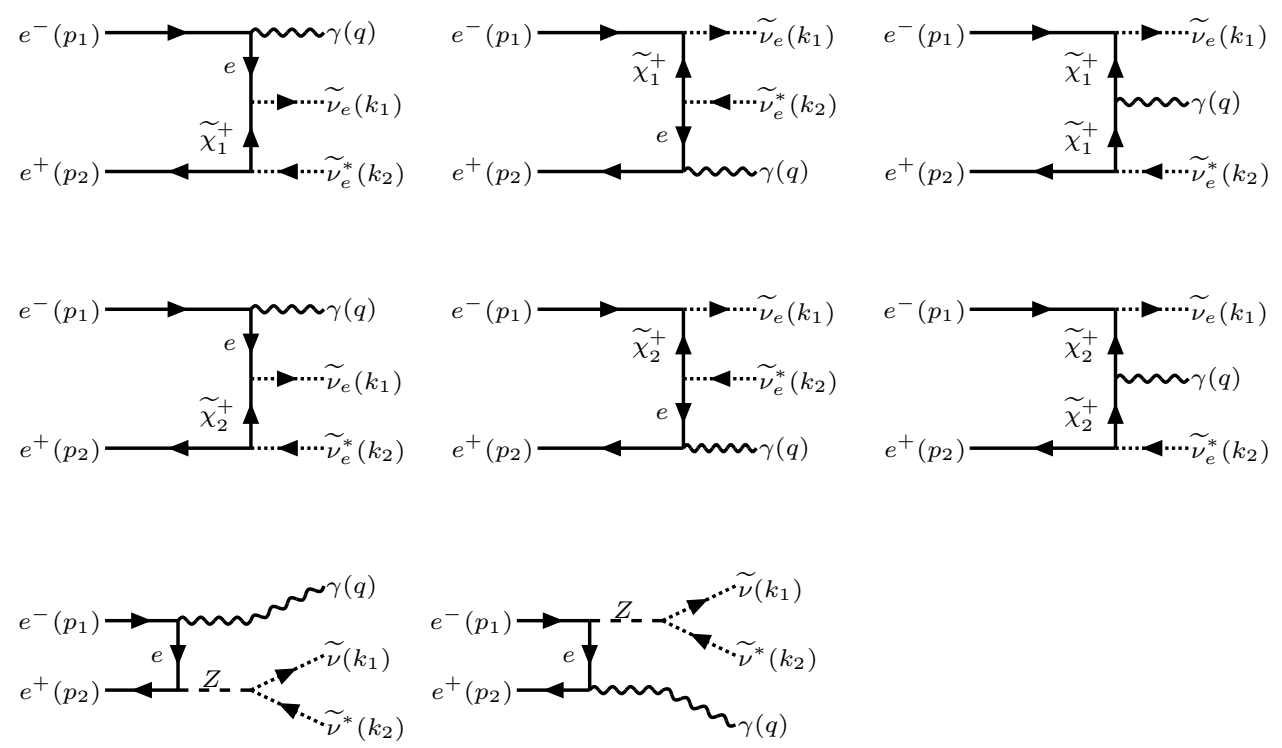

FIG. 15: Feynman diagrams contributing to the radiative sneutrino production process $e^{+} e^{-} \rightarrow \tilde{\nu} \tilde{\nu}^{*} \gamma$. 


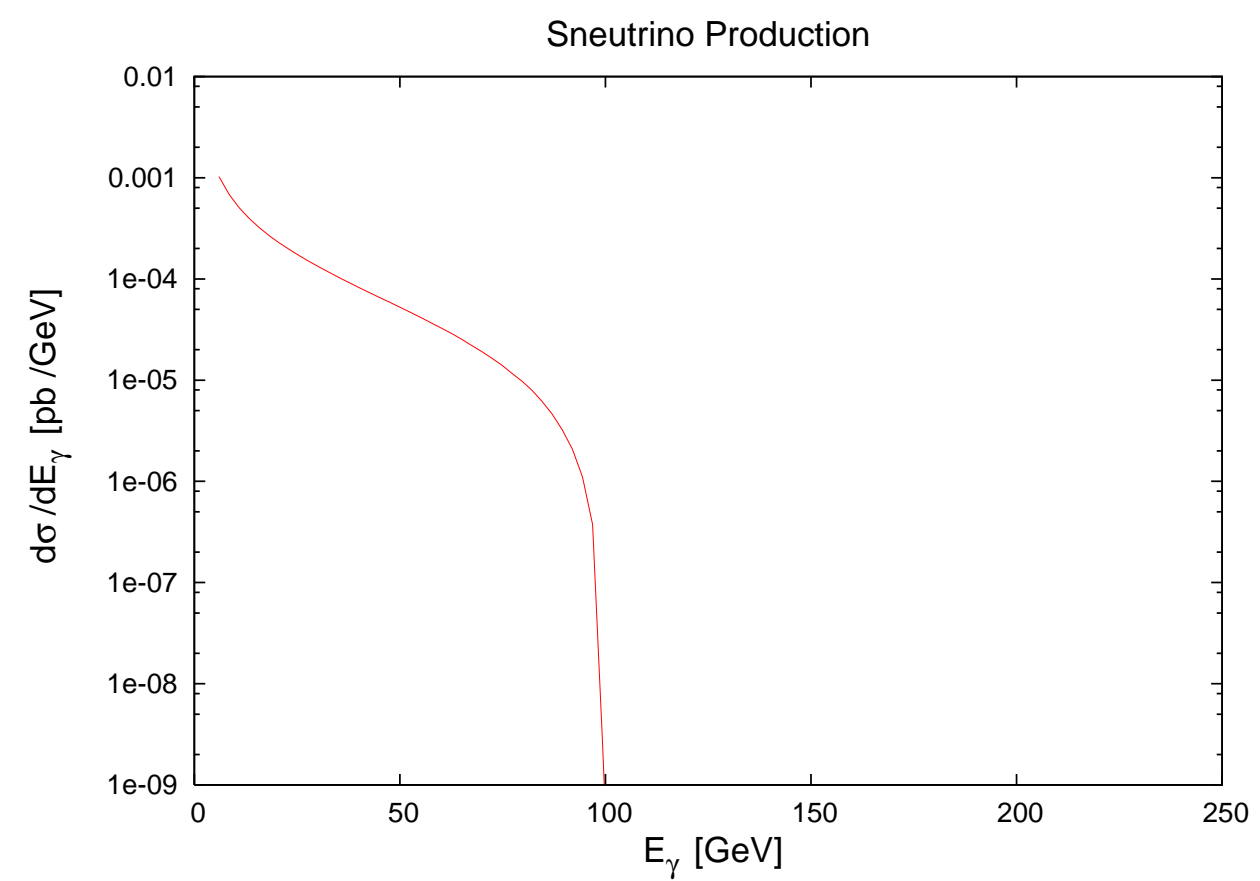

FIG. 16: The photon energy distribution $\frac{d \sigma}{d E_{\gamma}}$ for the radiative sneutrino production $e^{+} e^{-} \rightarrow \tilde{\nu} \tilde{\nu}^{*} \gamma$ at $\sqrt{s}=500 \mathrm{GeV}$.

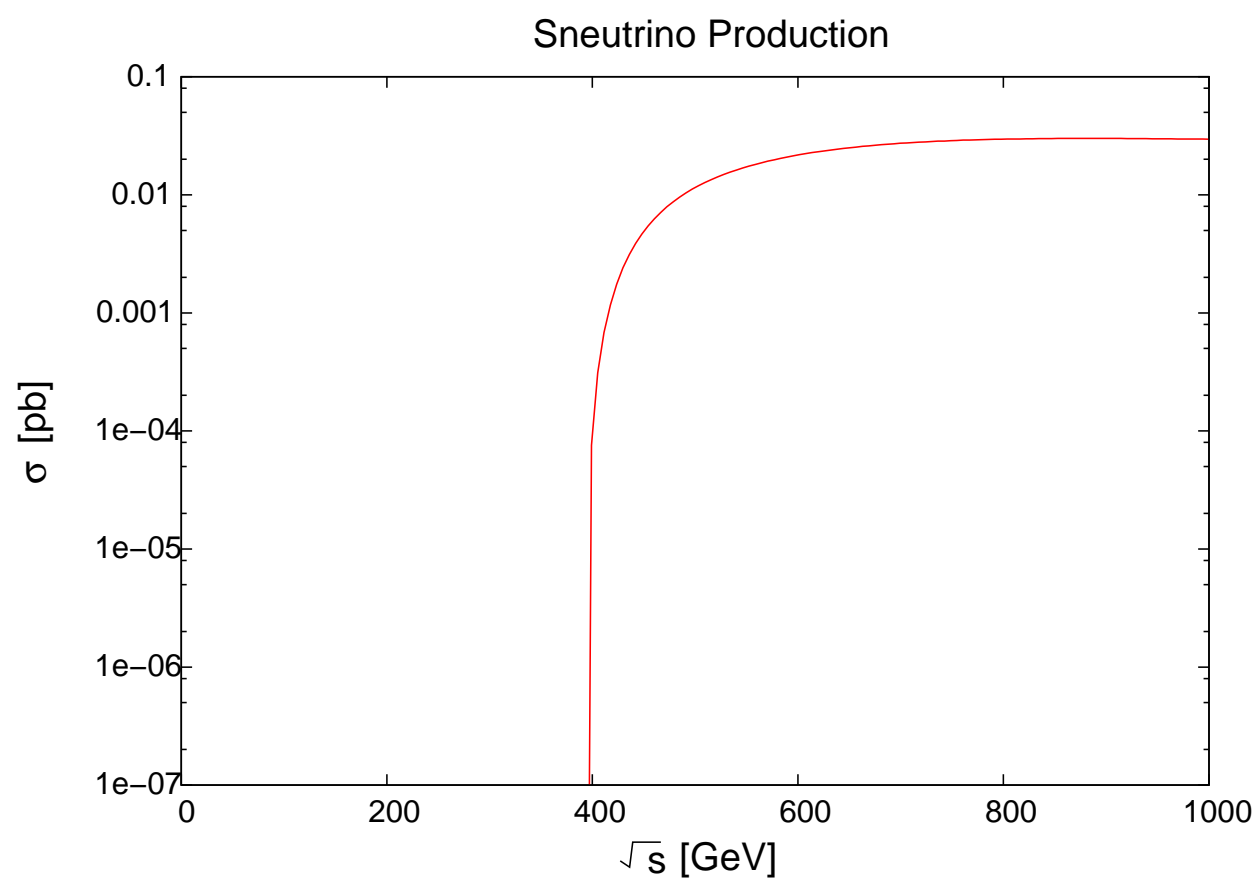

FIG. 17: Total energy $\sqrt{s}$ dependence of the radiative sneutrino production cross section $\sigma\left(e^{+} e^{-} \rightarrow \tilde{\nu} \tilde{\nu}^{*} \gamma\right)$.

\section{Theoretical Significance}

We now consider the question as to whether an excess of photons from radiative neutralino production can be measured over the SM background photons coming from radiative neutrino production. To quantify the excess of photons from the signal over the SM background photons for a given integrated luminosity $\mathcal{L}$, we 
Theoretical Significance(S) vs $\mu$

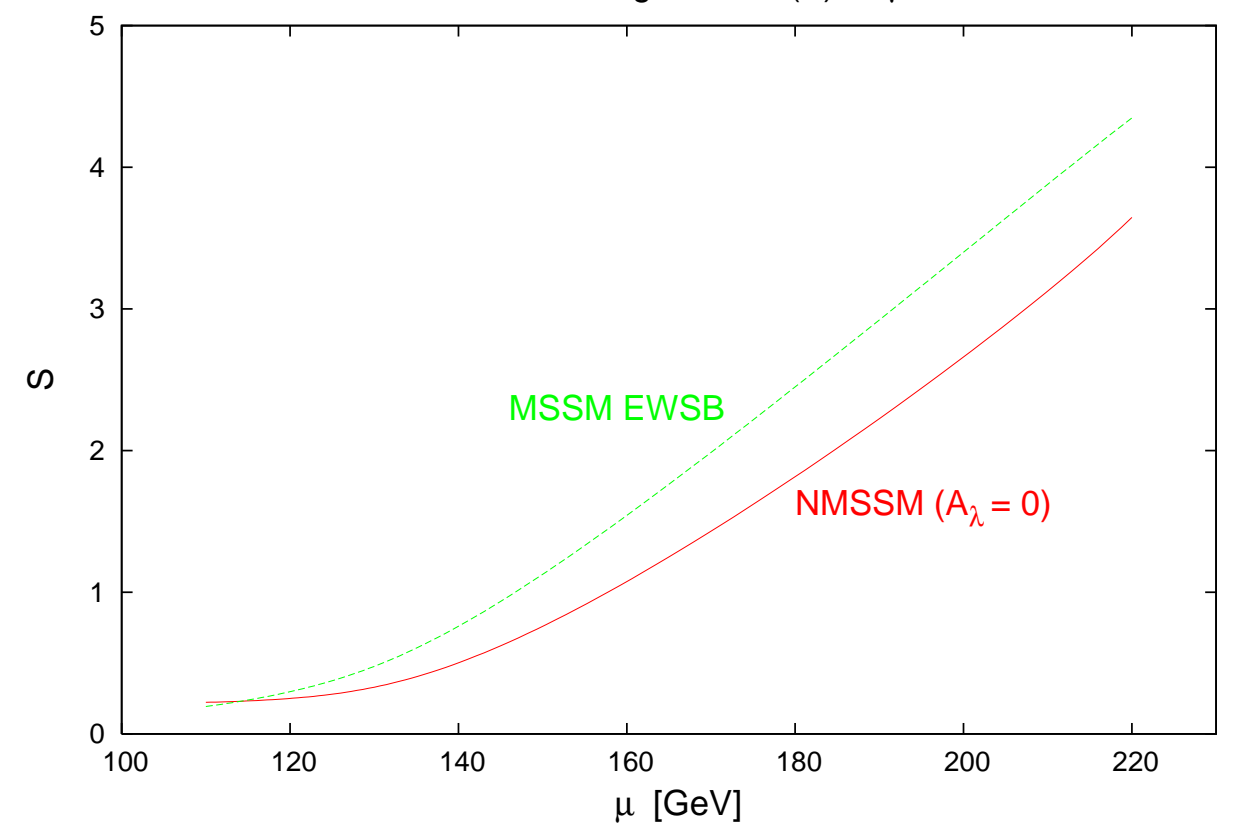

FIG. 18: Theoretical significance $S=\frac{\sigma}{\sqrt{\sigma+\sigma_{B}}} \sqrt{\mathcal{L}}$ for the radiative neutralino production versus $\mu$ for NMSSM (red solid line) and for MSSM in the EWSB scenario (green dashed). The value of $\sqrt{s}=500 \mathrm{GeV}$.

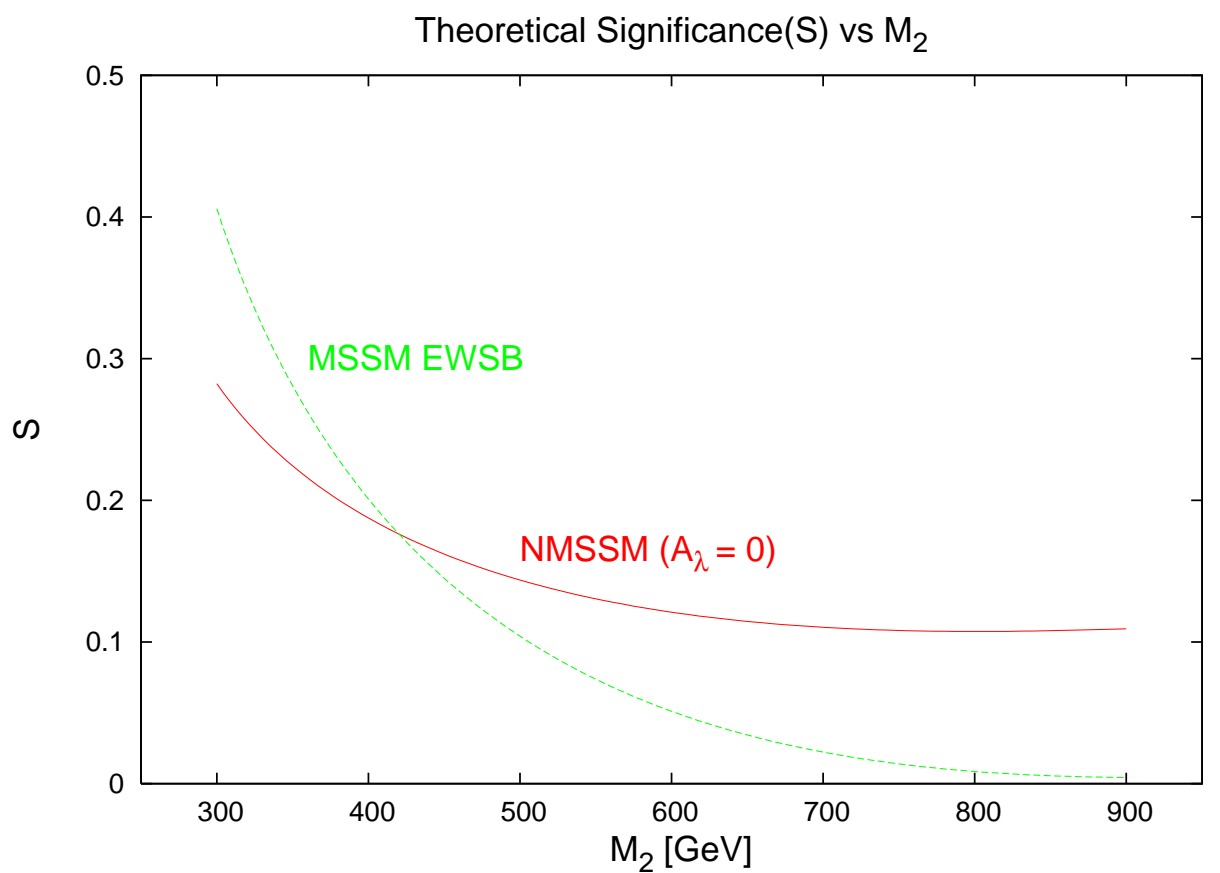

FIG. 19: Theoretical significance $S=\frac{\sigma}{\sqrt{\sigma+\sigma_{\mathrm{B}}}} \sqrt{\mathcal{L}}$ for the radiative neutralino production versus $M_{2}$ for NMSSM (red solid line) and for MSSM in the EWSB scenario (green dashed). The value of $\sqrt{s}=500 \mathrm{GeV}$.

consider the theoretical significance [50]

$$
S=\frac{N_{\mathrm{S}}}{\sqrt{N_{\mathrm{S}}+N_{\mathrm{B}}}}=\frac{\sigma}{\sqrt{\sigma+\sigma_{\mathrm{B}}}} \sqrt{\mathcal{L}}
$$

where $N_{\mathrm{S}}=\sigma \mathcal{L}$ is the number of signal photons, and $N_{\mathrm{B}}=\sigma_{\mathrm{B}} \mathcal{L}$ is the number of photons from the SM background of radiative neutrino production process. 
The processes $e^{+} e^{-} \rightarrow \tilde{\chi}_{1}^{0} \tilde{\chi}_{1}^{0} \gamma$ and $e^{+} e^{-} \rightarrow \nu \bar{\nu} \gamma$ depend significantly on the beam energy only near threshold in most of the parameter space for $\sqrt{s}=500 \mathrm{GeV}$ and $\mathcal{L}=5 \times 10^{5} \mathrm{pb}^{-1}$. In Fig. 18, we show the $\mu$ dependence of the theoretical significance $S$ for NMSSM. For this plot we have chosen $A_{\lambda}=0$, and varied the parameter $\mu$ in the interval $\mu \in[110,220] \mathrm{GeV}$. All other parameters for the NMSSM are chosen as in Table IV] For comparison we have also also shown in Fig. 18 the theoretical significance $S$ for the MSSM with parameters as in Table II for the EWSB model. We note that in NMSSM for $\mu<110 \mathrm{GeV}$, and with other parameters as described above, the Landau pole develops below the GUT scale, and the lightest chargino and the lightest neutralino masses are below the experimental lower bounds. On the other hand, for $\mu>220 \mathrm{GeV}$, the NMSSM develops unphysical global minima. We note from this analysis that Significance of $S \simeq 4$ can be attained for $\mu \simeq 220 \mathrm{GeV}$.

We have also studied the behavior of the theoretical significance as a function of the $S U(2)_{L}$ gaugino mass $M_{2}$. In Fig. 19. we show the $M_{2}$ dependence of the theoretical significance $S$ for NMSSM and MSSM in the interval $M_{2} \in[300,900] \mathrm{GeV}$. As in the study of the $\mu$ dependence, for NMSSM, we have chosen $A_{\lambda}=0$, and all other parameters as in Table IV] Furthermore, for the NMSSM the interval for $M_{2}$ is chosen in order to satisfy the theoretical and experimental constraints. For $M_{2}<300 \mathrm{GeV}$, the Landau pole for NMSSM develops below the GUT scale. On the other hand for values of $M_{2}>900$, there is a sfermion with negative mass squared in the spectrum of NMSSM. We note from Figs. 18 and 19 that higher values of $\mu$ and lower values of $M_{2}$ are favored for achieving higher values of the significance $S$. A theoretical significance of $S=1$ would mean that the signal can be measured at a $68 \%$ confidence level. On the other hand a significance of 5 is required for the detection of the signal. We note that a signal significance of about 4 can be achieved for NMSSM for values of $\mu \simeq 220 \mathrm{GeV}$. Besides the theoretical significance, one must also consider the signal to background ratio $N_{S} / N_{B}$ in order to judge the reliability of the analysis. It will be necessary to do a detailed Monte Carlo analyses to predict the significance. However, this is beyond the scope of the present work.

\section{SUMMARY AND CONCLUSIONS}

The nonminimal supersymmetric standard model is an attractive low energy supersymmetric model which solves the $\mu$ problem of the minimal supersymmetric standard model. We have carried out a detailed analysis of the radiative neutralino production $e^{+} e^{-} \rightarrow \tilde{\chi}_{1}^{0} \tilde{\chi}_{1}^{0} \gamma$ in the NMSSM for the International Linear Collider energies and compared it with the corresponding results in the MSSM. This process has a signature of a high energy photon and missing energy. We have obtained a typical set of parameter values for the NMSSM by imposing theoretical and experimental constraints on the parameter space of NMSSM. For the set of parameter values that we obtain in this manner, the lightest neutralino in NMSSM has a significant admixture of the fermionic component of the singlet chiral superfield $S$. Using this parameter set, we have studied in detail the radiative neutralino production cross section in NMSSM for the ILC energies with unpolarized $e^{+}$and $e^{-}$beams. For comparison, we have used the SPS 1a benchmark scenario for the MSSM. We have also calculated the background to this process from the SM process $e^{+} e^{-} \rightarrow \nu \bar{\nu} \gamma$, as well as the background from the supersymmetric process $e^{+} e^{-} \rightarrow \tilde{\nu} \tilde{\nu}^{*} \gamma$. All these processes have a signature of a highly energetic photon with missing energy. The photon energy distribution $d \sigma / d E_{\gamma}$, and the total cross section as a function of the total energy have been calculated for the NMSSM and for MSSM SPS 1a scenario at $\sqrt{s}=500 \mathrm{GeV}$ using CalcHEP. Because of the admixture of a singlet in the lightest neutralino, the cross section as a function of energy for the radiative neutralino production is much lower in NMSSM than in MSSM. We have also studied the dependence of the cross section for radiative neutralino production on the $S U(2)_{L}$ gaugino mass parameter $M_{2}$ and the Higgs(ino) mass parameter $\mu$, as well as its dependence on the selectron $\left(\tilde{e}_{R}, \tilde{e}_{L}\right)$ masses in NMSSM, and compared it with the corresponding results in MSSM. In order to quantify whether an excess of signal photons, $N_{\mathrm{S}}$, can be measured over the background photons, $N_{\mathrm{B}}$, from radiative neutrino production, we have analysed the theoretical statistical significance $S=N_{\mathrm{S}} / \sqrt{N_{\mathrm{S}}+N_{\mathrm{B}}}$, and studied its dependence on $M_{2}$ and $\mu$, the parameters that enter the neutralino mass matrix. The theoretical significance increases with the parameter $\mu$ for both NMSSM as well as MSSM, whereas it decreases as a function of $M_{2}$. The decrease is especially sharp in the case of minimal supersymmetric standard model. It may be interesting to study whether the signal for radiative neutralino production in NMSSM can be enhanced by using polarized beams [65].

\section{ACKNOWLEDGEMENTS}

P. N. P. would like to thank The Institute of Mathematical Sciences, Chennai, where this work was started, for its hospitality. He would also like to thank the Abdus Salam ICTP, where this work was completed, for 
its hospitality. The work of P. N. P. is supported by the Board of Nuclear Sciences, Department of Atomic Energy, India under project No. 2007/37/34/BRNS/1970. 
[1] J. Wess and J. Bagger, "Supersymmetry and supergravity," Princeton, USA: Univ. Pr. (1992) 259 p

[2] G. 't Hooft, in "Recent Developments In Gauge Theories. Proceedings, Nato Advanced Study Institute, Cargese, France, August 26 - September 8, 1979," New York, Usa: Plenum (1980) 438 P. (Nato Advanced Study Institutes Series: Series B, Physics, 59); E. Witten, Nucl. Phys. B 188, 513 (1981); R. K. Kaul, Phys. Lett. B 109, 19 (1982); R. K. Kaul and P. Majumdar, Nucl. Phys. B 199, 36 (1982); R. K. Kaul, Pramana 19, 183 (1982).

[3] H. P. Nilles, Phys. Rept. 110, 1 (1984).

[4] M. Drees, R. Godbole and P. Roy, "Theory and phenomenology of sparticles: An account of four-dimensional N=1 supersymmetry in high energy physics," Hackensack, USA: World Scientific (2004) 555 p.

[5] J. A. Aguilar-Saavedra et al. [ECFA/DESY LC Physics Working Group], "TESLA Technical Design Report Part III: Physics at an e+e- Linear Collider," arXiv:hep-ph/0106315.

[6] T. Abe et al. [American Linear Collider Working Group], "Linear collider physics resource book for Snowmass 2001. 1: Introduction," in Proc. of the APS/DPF/DPB Summer Study on the Future of Particle Physics (Snowmass 2001) ed. N. Graf, arXiv:hep-ex/0106055.

[7] K. Abe et al. [ACFA Linear Collider Working Group], "Particle physics experiments at JLC," arXiv:hep-ph/0109166

[8] G. Weiglein et al. [LHC/LC Study Group], "Physics interplay of the LHC and the ILC," arXiv:hep-ph/0410364.

[9] J. A. Aguilar-Saavedra et al., Eur. Phys. J. C 46, 43 (2006) arXiv:hep-ph/0511344.

[10] G. A. Moortgat-Pick et al., arXiv:hep-ph/0507011.

[11] P. Fayet, Nucl. Phys. B 90, 104 (1975); H. P. Nilles, M. Srednicki and D. Wyler, Phys. Lett. B 120, 346 (1983); J. P. Derendinger and C. A. Savoy, Nucl. Phys. B 237, 307 (1984).

[12] J. R. Ellis, J. F. Gunion, H. E. Haber, L. Roszkowski and F. Zwirner, Phys. Rev. D 39, 844 (1989).

[13] M. Drees, Int. J. Mod. Phys. A 4, 3635 (1989).

[14] P. N. Pandita, Phys. Lett. B 318, 338 (1993); P. N. Pandita, Z. Phys. C 59, 575 (1993); U. Ellwanger, Phys. Lett. B 303, 271 (1993) arXiv:hep-ph/9302224; U. Ellwanger, M. Rausch de Traubenberg and C. A. Savoy, Phys. Lett. B 315, 331 (1993) arXiv:hep-ph/9307322]; S. F. King and P. L. White, Phys. Rev. D 53, 4049 (1996) arXiv:hep-ph/9508346.

[15] B. Ananthanarayan and P. N. Pandita, Int. J. Mod. Phys. A 12, 2321 (1997) arXiv:hep-ph/9601372.

[16] B. Ananthanarayan and P. N. Pandita, Phys. Lett. B 371, 245 (1996) arXiv:hep-ph/9511415.

[17] B. Ananthanarayan and P. N. Pandita, Phys. Lett. B 353, 70 (1995) arXiv:hep-ph/9503323.

[18] M. Chemtob and P. N. Pandita, Phys. Rev. D 73, 055012 (2006) arXiv:hep-ph/0601159.

[19] M. Chemtob and P. N. Pandita, arXiv:0708.1284 [hep-ph].

[20] R. Dermisek and J. F. Gunion, Phys. Rev. Lett. 95, 041801 (2005) arXiv:hep-ph/0502105].

[21] U. Ellwanger, J. F. Gunion and C. Hugonie, JHEP 0507, 041 (2005) arXiv:hep-ph/0503203.

[22] G. A. Moortgat-Pick, S. Hesselbach, F. Franke and H. Fraas, In the Proceedings of 2005 International Linear Collider Workshop (LCWS 2005), Stanford, California, 18-22 Mar 2005, pp 0206 arXiv:hep-ph/0508313.

[23] J. F. Gunion, D. Hooper and B. McElrath, Phys. Rev. D 73, 015011 (2006) arXiv:hep-ph/0509024.

[24] P. N. Pandita, Phys. Rev. D 50, 571 (1994).

[25] P. N. Pandita, Z. Phys. C 63 (1994) 659.

[26] S. Y. Choi, D. J. Miller and P. M. Zerwas, Nucl. Phys. B 711, 83 (2005) arXiv:hep-ph/0407209.

[27] H. Goldberg, Phys. Rev. Lett. 50, 1419 (1983).

[28] J. R. Ellis, J. S. Hagelin, D. V. Nanopoulos, K. A. Olive and M. Srednicki, Nucl. Phys. B 238, 453 (1984).

[29] A. Bartl, H. Fraas and W. Majerotto, Nucl. Phys. B 278, 1 (1986).

[30] J. R. Ellis, J. M. Frere, J. S. Hagelin, G. L. Kane and S. T. Petcov, Phys. Lett. B 132, 436 (1983); E. Reya, Phys. Lett. B 133, 245 (1983); P. Chiappetta, J. Soffer, P. Taxil, F. M. Renard and P. Sorba, Nucl. Phys. B 262, 495 (1985), [Erratum-ibid. B 279, 824 (1987)].

[31] P. Fayet, Phys. Lett. B 117, 460 (1982).

[32] J. R. Ellis and J. S. Hagelin, Phys. Lett. B 122, 303 (1983).

[33] K. Grassie and P. N. Pandita, Phys. Rev. D 30, 22 (1984).

[34] T. Kobayashi and M. Kuroda, Phys. Lett. B 139, 208 (1984).

[35] J. D. Ware and M. E. Machacek, Phys. Lett. B 142, 300 (1984).

[36] L. Bento, J. C. Romao and A. Barroso, Phys. Rev. D 33, 1488 (1986).

[37] M. Chen, C. Dionisi, M. Martinez and X. Tata, Phys. Rept. 159, 201 (1988).

[38] T. Kon, Prog. Theor. Phys. 79, 1006 (1988).

[39] S. Y. Choi, J. S. Shim, H. S. Song, J. Song and C. Yu, Phys. Rev. D 60, 013007 (1999) arXiv:hep-ph/9901368.

[40] A. Datta, A. Datta and S. Raychaudhuri, Eur. Phys. J. C 1, 375 (1998) arXiv:hep-ph/9605432 ; A. Datta, A. Datta and S. Raychaudhuri, Phys. Lett. B 349, 113 (1995) arXiv:hep-ph/9411435.

[41] S. Ambrosanio, B. Mele, G. Montagna, O. Nicrosini and F. Piccinini, Nucl. Phys. B 478, 46 (1996) arXiv:hep-ph/9601292.

[42] A. Heister et al. [ALEPH Collaboration], Eur. Phys. J. C 28, 1 (2003).

[43] J. Abdallah et al. [DELPHI Collaboration], Eur. Phys. J. C 38, 395 (2005) arXiv:hep-ex/0406019.

[44] P. Achard et al. [L3 Collaboration], Phys. Lett. B 587, 16 (2004) arXiv:hep-ex/0402002].

[45] G. Abbiendi et al. [OPAL Collaboration], Eur. Phys. J. C 29, 479 (2003) arXiv:hep-ex/0210043.

[46] G. Abbiendi et al. [OPAL Collaboration], Eur. Phys. J. C 18, 253 (2000) arXiv:hep-ex/0005002. 
[47] K. J. F. Gaemers, R. Gastmans and F. M. Renard, Phys. Rev. D 19, 1605 (1979).

[48] D. Choudhury, H. K. Dreiner, P. Richardson and S. Sarkar, Phys. Rev. D 61, 095009 (2000) arXiv:hep-ph/9911365; A. Dedes, H. K. Dreiner and P. Richardson, Phys. Rev. D 65, 015001 (2002) arXiv:hep-ph/0106199; H. K. Dreiner, C. Hanhart, U. Langenfeld and D. R. Phillips, Phys. Rev. D 68, 055004 (2003) arXiv:hep-ph/0304289.

[49] M. Gataullin [LEP Collaboration], Eur. Phys. J. C 33, S791 (2004) arXiv:hep-ex/0311014.

[50] H. K. Dreiner, O. Kittel and U. Langenfeld, Phys. Rev. D 74, 115010 (2006) arXiv:hep-ph/0610020].

[51] B. C. Allanach et al., in Proc. of the APS/DPF/DPB Summer Study on the Future of Particle Physics (Snowmass 2001) ed. N. Graf, Eur. Phys. J. C25, 113 (2002) [eConf C010630, p125 (2001)] arXiv:hep-ph/0202233.

[52] H. E. Haber and G. L. Kane, Phys. Rept. 117, 75 (1985).

[53] A. Bartl, H. Fraas, W. Majerotto and N. Oshimo, Phys. Rev. D 40, 1594 (1989).

[54] A. Pukhov, arXiv:hep-ph/0412191.

[55] S. Eidelman et al. [Particle Data Group], Phys. Lett. B 592, 1 (2004).

[56] F. A. Berends, G. J. H. Burgers, C. Mana, M. Martinez and W. L. van Neerven, Nucl. Phys. B 301, 583 (1988).

[57] F. Boudjema et al., arXiv:hep-ph/9601224.

[58] G. Montagna, M. Moretti, O. Nicrosini and F. Piccinini, Nucl. Phys. B 541, 31 (1999) arXiv:hep-ph/9807465.

[59] F. Franke and H. Fraas, Phys. Rev. D 49, 3126 (1994).

[60] N. Ghodbane and H. U. Martyn, in Proc. of the APS/DPF/DPB Summer Study on the Future of Particle Physics (Snowmass 2001) ed. N. Graf, arXiv:hep-ph/0201233.

[61] H. E. Haber and D. Wyler, Nucl. Phys. B 323, 267 (1989).

[62] S. Ambrosanio and B. Mele, Phys. Rev. D 53, 2541 (1996) arXiv:hep-ph/9508237.

[63] S. Ambrosanio and B. Mele, Phys. Rev. D 55, 1399 (1997) [Erratum-ibid. D 56, 3157 (1997)] arXiv:hep-ph/9609212.

[64] H. Baer and T. Krupovnickas, JHEP 0209, 038 (2002) arXiv:hep-ph/0208277.

[65] R. Basu, P. N. Pandita and Chandradew Sharma, in preparation. 